\title{
RGDS-functionalized polyethylene glycol hydrogel-coated magnetic iron oxide nanoparticles enhance specific intracellular uptake by HeLa cells
}

\author{
This article was published in the following Dove Press journal: \\ International Journal of Nanomedicine \\ 10 April 2012 \\ Number of times this article has been viewed
}

\author{
Caner Nazli' \\ Tugba Ipek Ergenc ${ }^{2}$ \\ Yasemin Yar' \\ Havva Yagci Acar ${ }^{1,3}$ \\ Seda Kizilel',2 \\ 'Graduate School of Sciences \\ and Engineering, Koç University, \\ ${ }^{2}$ Department of Chemical and \\ Biological Engineering, College \\ of Engineering, Koç University, \\ ${ }^{3}$ Department of Chemistry, Faculty \\ of Arts and Sciences, Koç University, \\ Istanbul, Turkey
}

\begin{abstract}
The objective of this study was to develop thin, biocompatible, and biofunctional hydrogel-coated small-sized nanoparticles that exhibit favorable stability, viability, and specific cellular uptake. This article reports the coating of magnetic iron oxide nanoparticles (MIONPs) with covalently cross-linked biofunctional polyethylene glycol (PEG) hydrogel. Silanized MIONPs were derivatized with eosin Y, and the covalently cross-linked biofunctional PEG hydrogel coating was achieved via surface-initiated photopolymerization of PEG diacrylate in aqueous solution. The thickness of the PEG hydrogel coating, between 23 and $126 \mathrm{~nm}$, was tuned with laser exposure time. PEG hydrogel-coated MIONPs were further functionalized with the fibronectin-derived arginine-glycine-aspartic acid-serine (RGDS) sequence, in order to achieve a biofunctional PEG hydrogel layer around the nanoparticles. RGDS-bound PEG hydrogel-coated MIONPs showed a 17-fold higher uptake by the human cervical cancer HeLa cell line than that of amine-coated MIONPs. This novel method allows for the coating of MIONPs with nano-thin biofunctional hydrogel layers that may prevent undesirable cell and protein adhesion and may allow for cellular uptake in target tissues in a specific manner. These findings indicate that the further biofunctional PEG hydrogel coating of MIONPs is a promising platform for enhanced specific cell targeting in biomedical imaging and cancer therapy.

Keywords: PEG hydrogel, surface-initiated photopolymerization, nanoparticle encapsulation, agglomeration
\end{abstract}

\section{Introduction}

Nanoparticles are powerful candidates for current clinical diagnostics and therapeutic methods. Magnetic iron oxide nanoparticles (MIONPs) have a variety of applications in areas such as cancer and cardiovascular and neurological disorders. ${ }^{1}$ Specifically, MIONPs have been exploited widely in biotechnology and medicine as delivery systems for drugs, genes, and biomolecules; as imaging agents for the diagnosis of cancer; and for cell tracking and hyperthermia..$^{2-8}$ In addition to in vivo applications, MIONPs are useful for various in vitro medical applications such as the magnetic separation of cells, proteins, DNA/RNA, and other biomolecules. ${ }^{2}$ Although MIONPs find extensive use in biomedicine and in various in vitro applications, their clinical use has been hindered because of agglomeration and fast detection via immune cells in physiological medium. ${ }^{2,9}$ MIONPs without any surface modification exhibit hydrophobic surface properties with a large surface area to volume ratio. ${ }^{9-11}$ 
These particles tend to agglomerate and form large clusters, ${ }^{2,12}$ and because of this they are not appropriate for biomedical applications. ${ }^{2,9}$ Therefore, MIONPs for biomedical use are coated with an appropriate water-soluble organic materials to reduce or prevent agglomeration and to provide stability in physiological medium. Although some MIONPs have proper surface chemistry, they are prevented from reaching target tissue sites by some biological systems, such as the reticuloendothelial system, vascular endothelium, and blood-brain barrier. ${ }^{1}$ In order to overcome the problems associated with the tendency to aggregate and with fast blood clearance, biocompatible polymers such as polyethylene glycol (PEG) ${ }^{11}$ and polyvinylpyrrolidone ${ }^{12}$ are extensively exploited as encapsulating materials for MIONPs. Particularly, PEG-coated MIONPs demonstrate appropriate stability in a physiological environment. ${ }^{2}$ Previous studies have shown that the coating of nanoparticles with PEG increases half-life of nanoparticles in the body. ${ }^{2,9,13}$ It has also been observed that the properties of coating material influence magnetic field strength of MIONPs. For example, dextran coating decreased the magnetic field strength of MIONPs because of the decreased interaction between iron oxide cores and water molecules. ${ }^{14}$ Therefore, unique strategies are required to enhance the uptake of MIONPs by cancer cells specifically without altering their magnetic properties.

PEG hydrogels are the most widely exploited threedimensional, hydrophilic, synthetic polymeric networks because of their resemblance to natural living tissue, their biocompatibility, and their resistance to cell and protein adhesion in vivo. ${ }^{15-17}$ PEG is approved by the US Food and Drug Administration as a material with extensive use in biomaterials, biotechnology, and medicine. ${ }^{17-19}$ Because of these desirable properties, PEG and PEG hydrogels are commonly used for applications in tissue engineering as extracellular matrix mimics, or as the coating material for encapsulation of cells and nanoparticles. ${ }^{17,20-23}$ Hydrogelencapsulated nanoparticles have created a tremendous interest because of their unique properties and broad applications, although conventional hydrogels have limited applications because of their poor mechanical strength. ${ }^{24,25}$ Recently, PEG hydrogel nanocomposite has been investigated as a potential implantable biomaterial for thermal cancer therapy applications. ${ }^{22}$ The preparation of hydrogel nanocomposite involves incorporation of specific nanoparticle material within a hydrogel matrix in order to enhance properties of hydrogels or nanoparticles. Thus far, various nanoparticle-hydrogel composite systems comprising metallic nanoparticles, carbon nanotubes, clays, ceramics, magnetic nanoparticles, hydroxyapatite, or semiconducting nanoparticles have been reported. ${ }^{26-30}$ One of the advantages of hydrogel encapsulation of nanoparticles is enhanced biocompatibility achieved through the presence of a hydrogel matrix between sensitive tissues and potentially harmful nanoparticles. For example, a recent study by Meenach et $\mathrm{a}^{22}$ demonstrated more favorable cell viability for iron oxide nanoparticles encapsulated within poly- $N$-isopropylacrylamide hydrogel than for unencapsulated nanoparticles.

Previous experimental studies with nanoparticle-hydrogel composite systems were based on bulk polymerization or incorporation of nanoparticles within an existing hydrogel matrix. Experimental efforts involving nanoencapsulation or coating of nanoparticles within a biofunctional PEG hydrogel capsule do not yet exist. This work reports, for the first time in the literature, the surface-initiated photopolymerization technique used for the encapsulation of single MIONPs or small clusters within a biofunctional PEG hydrogel. The objective of this study was to develop thin, biocompatible, and biofunctional hydrogel-coated smallsized nanoparticles that exhibit favorable stability, viability, and specific cellular uptake. In order to achieve the individual or cluster coating of nanoparticles with a nano-thin PEG hydrogel layer via surface-initiated photopolymerization, we applied a technique previously developed by Kizilel et $\mathrm{al}^{18,31-34}$ (Figure 1A). PEG hydrogel was functionalized with arginine-glycine-aspartic acid-serine (RGDS) in order to achieve binding of the PEG hydrogel-coated MIONP structure to $\alpha_{\mathrm{v}} \beta_{3}$ integrin, which is located at the outer cell membrane of the tumor endothelial cells. ${ }^{1,35,36}$ Biofunctional PEG hydrogel-encapsulated MIONPs in a core-shell structure have a nanometer-sized diameter, which is composed of a magnetic core, a cross-linked PEG shell, and covalently bound RGDS domains. We hypothesized that an RGDS-functionalized PEG hydrogel coating may increase the efficiency of internalization of MIONPs at the tumor site and prevent agglomeration and cell and protein adhesion. ${ }^{19}$ Another advantage of the method used here involves synthesis of small-sized and stable MIONPs through a 3-aminopropyltrimethoxysilane (APTMS) coating, which enables ultrasmall MIONPs to be coated with PEG hydrogel and the covalent attachment of APTMS to the iron oxide surface, which enables a stable coating and foundation for photopolymerization.

After surface-modification steps, MIONPs were characterized using Fourier transform infrared (FT-IR) spectroscopy, dynamic light scattering (DLS), zeta potential 
A
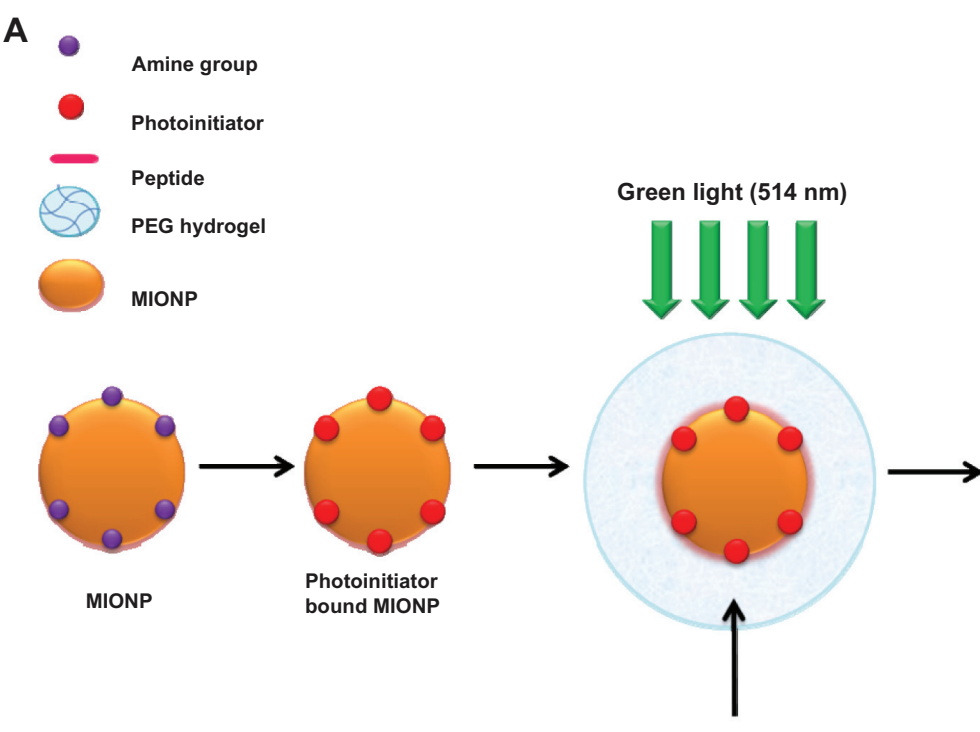

Prepolymer solution

\section{B}
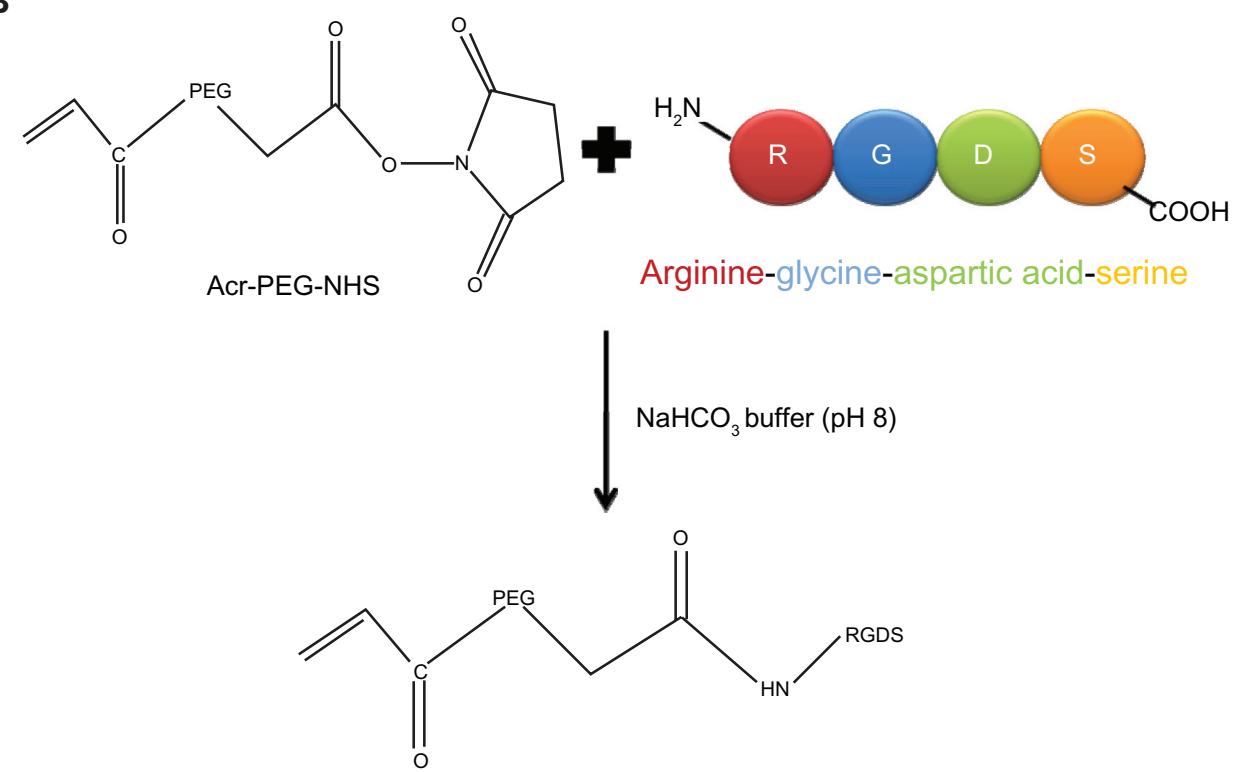

Acr-PEG-RGDS

Figure I (A) Schematic representation of the coating of an iron oxide nanoparticle with biofunctional polyethylene glycol (PEG) hydrogel via surface-initiated photopolymerization; (B) schematic representation of acrylate (Acr)-PEG-arginine-glycine-aspartic acid-serine (RGDS) synthesis.

Abbreviation: MIONP, magnetic iron oxide nanoparticle.

measurement, scanning electron microscopy (SEM), and atomic force microscopy (AFM). HeLa cells that were incubated with RGDS-functionalized PEG hydrogel-coated MIONPs demonstrated enhanced viability compared with those exposed to APTMS-coated or only PEG hydrogelcoated MIONPs. Further, the biofunctional PEG hydrogelcoated MIONP system was superior in terms of intracellular uptake compared to other systems containing APTMS-coated or only PEG hydrogel-coated nanoparticles, as shown via
Prussian blue staining and inductively coupled plasma optical emission spectrometry (ICP-OES).

\section{Material and methods Materials}

$\mathrm{FeCl}_{3} \cdot 6 \mathrm{H}_{2} \mathrm{O}$ and $\mathrm{FeCl}_{2} \cdot 4 \mathrm{H}_{2} \mathrm{O}$ were used as purchased from Fluka (Sigma-Aldrich, Taufkirchen, Germany). Ammonium hydroxide $\left(26 \% \mathrm{NH}_{3}\right.$ in water, w/w) was purchased from Riedel-de Haën (Hanover, Germany). APTMS was purchased 
from Gelest, Inc (Morrisville, PA). Eosin Y (91\%), 1-vinyl2-pyrrolidinone (VP; 99\%), PEG diacrylate (PEGDA) (MW: $575 \mathrm{Da}$ ), Woodward's reagent K(WRK) (2-ethyl-5-phenylisoxazolium-3'-sulfonate, 95\%), phosphate-buffered saline (PBS), and Triton ${ }^{\circledR}$ X-100 were purchased from Sigma-Aldrich. Triethanolamine (TEA) (99.5\%), sodium bicarbonate, and 37\% formaldehyde were obtained from Merck (Whitehouse Station, $\mathrm{NJ})$. Hydrochloric acid $(\mathrm{HCl})$ and sodium hydroxide were purchased from Riedel-de Haën. The Amicon Ultra centrifugal filter device (NMWL: 3000) was obtained from EMD Millipore (Billerica, MA). Short peptide RGDS (MW: 433 Da) was purchased from Elim Biopharmaceuticals, Inc (Hayward, CA). Acrylate PEG $N$-hydroxysuccinimide (acryl-PEG-NHS) (MW: $3.4 \mathrm{kDa}$ ) was purchased from Nektar (Huntsville, AL). The dialysis cassette (MW cutoff: $3.5 \mathrm{kDa}$ ) and bicinchoninic acid protein assay were purchased from Pierce (Micro BCA Protein Assay; Thermo Fisher Scientific Inc, Rockford, IL). Teflon syringe filters $(0.2 \mu \mathrm{m}$ pore size $)$ were obtained from Nalgene (Rochester, NY). Petri dishes were purchased from Nunc (Roskilde, Denmark). Dulbecco's modified Eagle medium (liquid, with $4.5 \mathrm{~g} / \mathrm{L} \mathrm{D}$-glucose and sodium pyruvate) (DMEM), L-glutamine, and penicillin/streptomycin were purchased from Invitrogen Co (Carlsbad, CA). Fetal bovine serum was obtained from Biological Industries (Rainbow Scientific, Inc, Windsor, CT). Trypsin-ethylenediaminetetraacetic acid was purchased from Genlantis, Inc (San Diego, CA). CellTiter-Glo ${ }^{\circledR}$ Luminescent Cell Viability Assay Kit was obtained from Promega (Madison, WI). Cell culture plates (96- and six-well) were purchased from Greiner Bio-One GmbH (Solingen, Germany). Ninety-six-well LUMITRAC ${ }^{\circledR}$ 600 white immunology plates were obtained from USA Scientific, Inc (Ocala, FL). Microscope slides were purchased from Fisher Scientific (CA). Cover slides were obtained from Isolab. Potassium ferrocyanide was purchased from Labkim (Istanbul, Turkey).

\section{Synthesis of acryl-PEG-RGDS conjugate}

The synthesis of acryl-PEG-RGDS conjugate was performed as represented in Figure 1B. Short peptide RGDS $(1 \mathrm{mg} / \mathrm{mL})$ solution and acryl-PEG-NHS (10 mg/mL) solution were prepared separately in $50 \mathrm{mM}$ of sodium bicarbonate at $\mathrm{pH}$ 8.2. Acryl-PEG-NHS solution was added drop-wise to the peptide solution until the mole ratio of acryl-PEG-NHS to peptide was $1: 1$. The conjugation reaction was carried out for 2 hours at room temperature and with continuous rocking. After the reaction, the reaction mixture was dialyzed against deionized water in order to remove unreacted acryl-PEG-NHS and peptide.
The amount of acryl-PEG-RGDS in the conjugate product was determined by bicinchoninic acid protein assay using a NanoDrop 1000 spectrophotometer (Fisher Scientific (Austria) GmbH, Vienna, Austria) set at a wavelength of $562 \mathrm{~nm}$. The conjugate product included 70\% acryl-PEGRGDS. The binding of RGDS peptide to acryl-PEG-NHS was confirmed by FT-IR spectroscopy (Nicolet iS10 FT-IR spectrometer; Fisher Scientific (Austria) GmbH).

\section{Synthesis of APTMS-coated MIONPs}

Milli-Q ${ }^{\circledR}$ (EMD Millipore) water $(45 \mathrm{~mL})$ was deoxygenated for 30 minutes in a $100 \mathrm{~mL}$ three-necked round-bottom flask fitted with a mechanical stirrer and a condenser. Iron salts $\left(\mathrm{Fe}^{3+} / \mathrm{Fe}^{2+}\right.$ mole ratio, $=2$; total iron concentration, $\left.0.175 \mathrm{M}\right)$ were added to the flask and stirred at $400 \mathrm{rpm}$ under nitrogen for about 15 minutes. The reaction flask was placed into an oil bath at $85^{\circ} \mathrm{C}$. After 10 minutes of mixing, APTMS ( $\mathrm{Si} / \mathrm{Fe}$ mole ratio, 1.25) and excess amount of ammonium hydroxide were injected into the flask with vigorous stirring at $600 \mathrm{rpm}$. The reaction was allowed to continue for 1 hour to produce a stable colloidal solution; then it was cooled to room temperature and placed on top of a magnet ( 0.3 Tesla) for a few hours. Any precipitate was removed with magnetic decantation. Nanoparticles were washed using the Amicon Ultra centrifugal filter with deionized water. The amount of organic coating on MIONPs was calculated through thermogravimetric analysis (TGA 50/50H, Shimadzu Deutschland $\mathrm{GmbH}$, Duisburg, Germany) performed on dried powders.

\section{Eosin binding onto the surface of MIONPs}

Eosin solution $(4 \mathrm{mM})$ in deionized water and WRK $(20 \mathrm{mM})$ solution in sodium hydroxide $(0.1 \mathrm{M})$ were prepared and the solutions were adjusted to $\mathrm{pH}$ 9. Equal volumes of both the eosin and the WRK solution were mixed and reacted for 30 minutes. Equal volumes $(10 \mathrm{~mL})$ of $40 \mathrm{mg} / \mathrm{mL}$ aqueous APTMS-coated MIONPs and eosin-WRK mixture were reacted overnight at room temperature and at $\mathrm{pH} 9$ in order to form amide bonds between the carboxylic acid group of eosin and the amine group of APTMS-coated MIONPs (final reaction solution contained $20 \mathrm{mg} / \mathrm{mL}$ APTMS-coated MIONPs, $1 \mathrm{mM}$ of eosin and $5 \mathrm{mM}$ of WRK) (Figure 2A). Next, the eosin-bound MIONP solution was washed using the Amicon Ultra centrifugal filter with deionized water in order to remove unbound eosin, excess WRK, and other ions. The amount of eosin on the MIONPs was determined by the absorbance at $537 \mathrm{~nm}$, using the Nanodrop 1000 spectrophotometer. 
A

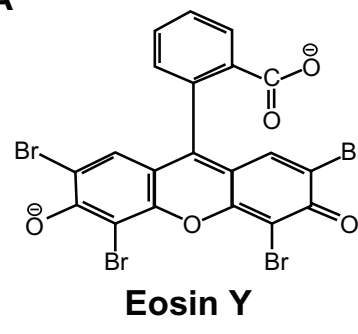<smiles>CCO[Si](CCCN)(OCC)OOC1CCCCCCCCC1</smiles>

WRK (pH 8)

APTMS coated MIONPS<smiles>CCOS(CC)(CCCNC(=O)c1ccccc1C1=C(Br)C(O)C(Br)=C(Br)O1)OC1CCCCCCCCCCC1</smiles>

Eosin bound MIONP

B

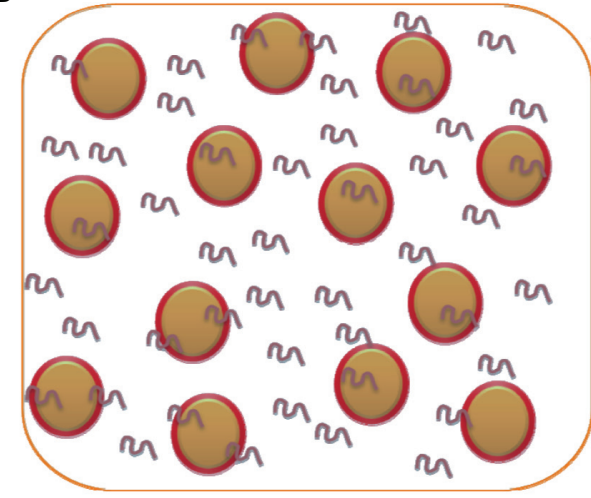

Eosin bound MIONPs dispersed in prepolymer solution

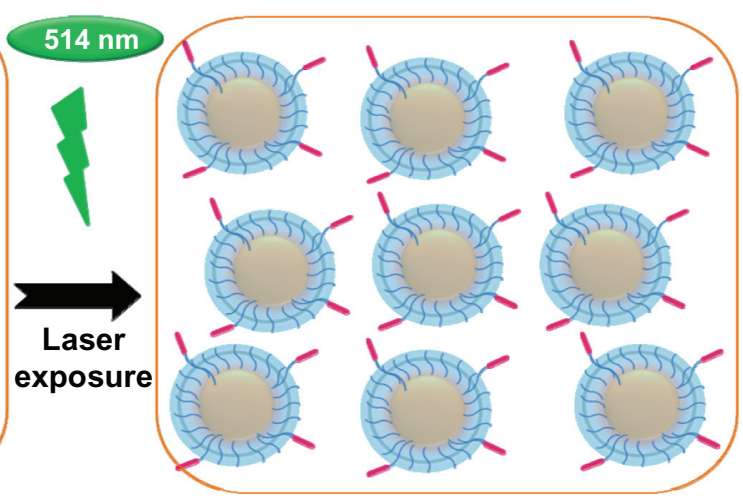

Biofunctional PEG hydrogel coated MIONP

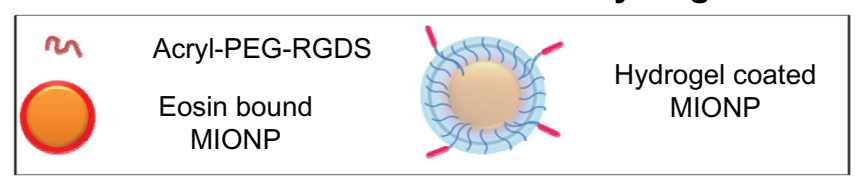

Figure 2 (A) Schematic representation of eosin binding onto the surface of amine-functionalized magnetic iron oxide nanoparticles (MIONPs); (B) encapsulation of eosinbound MIONPs within arginine-glycine-aspartic acid-serine (RGDS)-functionalized polyethylene glycol (PEG) hydrogel.

Abbreviations: Acryl, acrylate; APTMS, 3-aminopropylsilane; WRK, Woodward's reagent $\mathrm{K}$.

\section{Prepolymer solution preparation}

Prepolymer solution (PPS) containing 6.25\% PEGDA (w/v) (MW: $575 \mathrm{Da})$, TEA $(141 \mathrm{mM})$, and VP $(24 \mathrm{mM})$ was prepared in deionized water. The solution was adjusted to $\mathrm{pH} 8$ using $\mathrm{HCl}(6 \mathrm{M})$. In order to produce an RGDS-functionalized PEG hydrogel coating around MIONPs, acryl-PEG-RGDS was added to the PPS at a final concentration of $1.48 \times 10^{-5} \mathrm{M}$.

\section{Hydrogel coating of MIONPs}

The eosin-bound MIONP solution was mixed with the PPS in order to achieve a final concentration of $0.1 \mathrm{mg} / \mathrm{mL}$ eosin-bound MIONPs, 5\% PEGDA, $19 \mathrm{mM}$ of VP, $113 \mathrm{mM}$ of TEA, and $1.48 \times 10^{-5} \mathrm{M}$ acryl-PEG-RGDS (for RGDSfunctionalized hydrogel-coated nanoparticles). The solution was adjusted to $\mathrm{pH} 8$. Next, the mixture was illuminated with 
green light at $514 \mathrm{~nm}$ and a flux of $2.5 \mathrm{~mW} / \mathrm{cm}^{2}$ using an argon ion laser (Coherent Inc, Santa Clara, CA) at various exposure times (20, 30, and 60 seconds) (Figure 2B). In order to remove unreacted PEGDA, acryl-PEG-RGDS, and other unreacted components, coated MIONPs were washed using the Amicon Ultra centrifugal filter. The washed coated MIONPs were stored at $4^{\circ} \mathrm{C}$.

\section{Structural, chemical, and physicochemical characterization of MIONPs}

APTMS-coated, eosin-bound, and PEG hydrogelencapsulated MIONPs with or without RGDS were characterized in terms of structure, size, stability, and morphology. In order to investigate the crystal structure of APTMS-coated MIONPs and PEG hydrogel-coated MIONPs, X-ray diffraction (XRD) was performed (Bruker AXS D8 Advance; Karlsruhe, Germany). FT-IR spectroscopy was used to follow changes in the chemical structure after each coating step. The hydrodynamic size of particles was determined using DLS at room temperature (Malvern zetasizer nano S ZEN 1600, wavelength $633 \mathrm{~nm}$; Malvern Instruments, Malvern, UK). The colloidal stability of nanoparticles was characterized through zeta potential analysis using a ZetaPALS (Brookhaven Instruments Corporation, Holtsville, NY). PEG hydrogel-coated MIONPs were physically adsorbed on a silicon surface and dried at $50^{\circ} \mathrm{C}$ for 1 hour, and the morphology of PEG hydrogel-coated MIONPs was analyzed via SEM (Zeiss Ultra Plus, Bruker; Optronik Ltd Sti, Ankara, Turkey) and the SOLVER S47 scanning probe microscopy platform (NT-MDT Europe BV, Eindhoven, The Netherlands) in intermittent AFM mode.

\section{Evaluation of cytotoxicity of nanoparticles}

The effect of nanoparticles on cell viability was investigated using HeLa cells. Cells were seeded at $5 \times 10^{4}$ cells $/ \mathrm{mL}$ in a 96-well plate and cultured for 24 hours in DMEM (pH 7.4, with $10 \%$ fetal bovine serum, 1\% penicillin/ streptomycin, and L-glutamine). Suspensions with APTMS-coated MIONPs, eosin-bound MIONPs, PEG hydrogel-coated MIONPs, and RGDS-functionalized PEG hydrogel-coated MIONPs were added to the HeLa cell culture medium at separate concentrations of $0.01,0.05$, and $0.1 \mathrm{mg} / \mathrm{mL}$. After 24 and 48 hours of incubation, the CellTiter-Glo Luminescent Viability Assay was carried out using a Fluoroskan Ascent luminescence reader (Fisher Scientific (Austria) GmbH).

\section{Characterization of internalization of nanoparticles by Prussian blue staining}

Intracellular uptake of APTMS, PEG hydrogel-coated MIONPs, and RGDS-functionalized PEG hydrogel-coated MIONPs were characterized via Prussian blue staining. HeLa cells $\left(5 \times 10^{6}\right)$ were cultured for 24 hours in six-well plates on glass coverslips. After uniform attachment of HeLa cells on the slide surface was achieved, nanoparticles at a concentration of $0.1 \mathrm{mg} / \mathrm{mL}$ were added into each well. After 24 hours of incubation, the HeLa cells on the cover slides were washed three times with PBS and fixed with $4 \%$ formaldehyde for 20 minutes at room temperature. After fixation the cells were treated with $0.1 \%$ Triton X-100 for 5 minutes and rinsed with PBS for permeabilization, and this step was repeated four times. Next, fixed HeLa cells were incubated in 5\% potassium ferrocyanide and $10 \% \mathrm{HCl}$ for 15 minutes. Finally, iron staining was observed via a camera equipped for optical microscopy (Motic DM-B1 Optical Microscope, Motic, Wetzlar, Germany).

\section{Determination of intracellular iron content}

Cellular uptakes of nanoparticles were determined qualitatively via ICP-OES. HeLa cells were grown in DMEM in a 24-well plate $\left(10^{5}\right.$ cells in $1 \mathrm{~mL}$ of medium; $1 \mathrm{~mL}$ of cell culture was added into each well). After 24 hours of incubation, APTMS, PEG hydrogel-coated and RGDS-functionalized PEG hydrogel-coated MIONPs were added into the medium at a concentration of $0.1 \mathrm{mg} / \mathrm{mL}$. In control samples the cells were placed in medium $(1 \mathrm{~mL})$ without nanoparticles by using the same cell density. After 24 hours of treatment of cells with nanoparticles, cells were rinsed three times with PBS. Cells were detached from the surface via trypsin-ethylenediaminetetraacetic acid solution $(0.25 \mathrm{~mL}$ in each well). Detached cells were incubated in a mixture of $65 \%$ nitric acid $(0.25 \mathrm{~mL})$ and $30 \%$ hydrogen peroxide $(0.25 \mathrm{~mL})$ for 24 hours at $90^{\circ} \mathrm{C}$. Finally, ICP-OES analysis was carried out for all samples, using an inductively coupled plasma optical emission spectrometer (SPECTRO GENESIS; SPECTRO Analytical Instruments $\mathrm{GmbH}$, Kleve, Germany). Reported values were averages of three runs and three samples.

\section{Statistical analysis}

The results of all data sets were analyzed using one-way analysis of variance. The results are represented as the mean value (plus or minus standard deviation) of the triplicate samples unless otherwise stated. Differences between datasets are considered statistically significant for $P$-values less than 0.05 . 


\section{Results}

\section{Synthesis of acryl-PEG-RGDS conjugate}

Covalent conjugation of RGDS peptide with acryl-PEG-NHS was confirmed with FT-IR spectroscopy. Carbonyl groups $(\mathrm{C}=\mathrm{O})$ in NHS ester exhibit two peaks at $1781 \mathrm{~cm}^{-1}$ and $1820 \mathrm{~cm}^{-1} \cdot{ }^{37}$ After conjugation reaction, the disappearance of these carbonyl bands confirms that NHS ester reacted with the amine groups in the RGDS structure, and new amide bonds were formed (Figure S1). The peak corresponding to the carbonyl group in acryl-PEG-NHS was observed around $1740 \mathrm{~cm}^{-1}$. After the conjugation reaction, the amide I peak appeared at $1670 \mathrm{~cm}^{-1} .38$ This new peak is clear evidence for the presence of RGDS in acryl-PEG-RGDS conjugate.

\section{Synthesis and characterization of MIONPs}

Stable and ultrasmall (6.4 nm in hydrodynamic size) aqueous MIONPs were synthesized with $20 \%$ (w/w) of APTMS coating. The XRD pattern of the APTMS-coated iron oxide nanoparticles exhibit a typical six-peak pattern ${ }^{39}$ that fits well with a maghemite-like structure - more specifically, $\mathrm{Fe}_{2.67} \mathrm{O}_{4}$ (Figure 3A). Ultraviolet and visible (UV-Vis) spectra of APTMScoated and eosin-bound MIONPs (washed) are provided in Figure S2A. The appearance of a new peak in the UV-Vis spectra of eosin-bound MIONPs around $530 \mathrm{~nm}$ confirmed eosin binding on APTMS-coated MIONPs. Absorbance of eosin-bound MIONPs at $530 \mathrm{~nm}$ indicated $0.57 \mathrm{mM}$ eosin concentration on the nanoparticle surface. ${ }^{40}$ This value corresponds to $57 \%$ efficiency in eosin binding to the MIONP surface.

The binding of eosin onto the surface of APTMS-coated MIONPs was also confirmed by FT-IR spectroscopy analysis. Figure S2B shows FT-IR spectra of both APTMS-coated

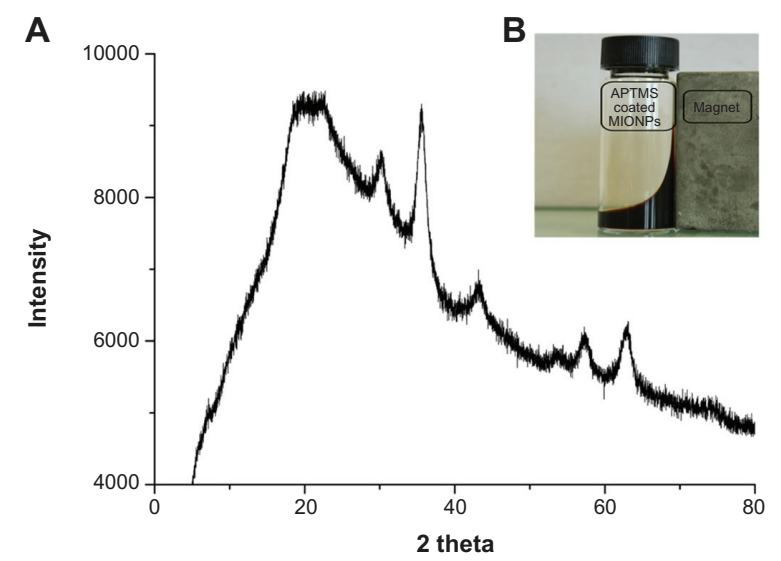

Figure 3 (A) X-ray diffraction patterns of 3-aminopropylsilane (APTMS)-coated magnetic iron oxide nanoparticles (MIONPs); (B) ferrofluid under an external magnetic field. and eosin-bound MIONPs. MIONPs exhibited two peaks at $571 \mathrm{~cm}^{-1}$ and $590 \mathrm{~cm}^{-1}$ because of the iron oxide cores. ${ }^{41-43}$ The peaks at $919,1215,1328,1388,1505,1617$, and $2931 \mathrm{~cm}^{-1}$ were assigned to $\mathrm{C}-\mathrm{H}$ out-of-plane bending, aliphatic $\mathrm{C}-\mathrm{N}$ stretching, aliphatic $\mathrm{C}-\mathrm{O}$ stretching, $\mathrm{Si}-\mathrm{CH}_{2}$ scissoring, $\mathrm{C}-\mathrm{H}$ bending, $\mathrm{NH}_{2}$ scissoring and $\mathrm{N}-\mathrm{H}$ bending, and $\mathrm{CH}_{2}$ stretching, respectively. ${ }^{41,44,45}$ After eosin binding, new bands that appeared at $1456 \mathrm{~cm}^{-1}$ and $1541 \mathrm{~cm}^{-1}$ corresponded to aromatic $\mathrm{C}=\mathrm{C}$ stretching of eosin. These new peaks overlapped with the $\mathrm{C}-\mathrm{H}$ bending peak at $1505 \mathrm{~cm}^{-1}$. The peaks at 3058 and $3352 \mathrm{~cm}^{-1}$ belonged to $\mathrm{NH}_{2}$ stretching of the APTMS-coated MIONPs. ${ }^{45}$ This feature disappeared to a great extent, and one new peak at $3156 \mathrm{~cm}^{-1}$ that corresponded to secondary amide $\mathrm{N}-\mathrm{H}$ stretching appeared after the eosin binding. ${ }^{45}$ These two FT-IR spectra gave strong evidence about covalent binding of eosin to the amine groups of APTMS-coated MIONPs.

After the photopolymerization reaction, characteristic eosin absorbance at $517 \mathrm{~nm}$ disappeared in the UV-Vis spectra of PEG hydrogel-coated MIONPs (Figure S3). This is expected as a result of eosin bleaching during light exposure. The presence of PEG and RGDS peptides on MIONPs was shown with FT-IR spectra (Figure 4). After PEG hydrogel coating, new bands seen at 1100 and $1352 \mathrm{~cm}^{-1}$ indicated symmetric and antisymmetric $\mathrm{C}-\mathrm{O}-\mathrm{C}$ stretching bands, respectively. The peaks at 1724 and $2850 \mathrm{~cm}^{-1}$ were assigned to $\mathrm{C}-\mathrm{H}$ out-of-plane bending, $\mathrm{C}=\mathrm{O}$ stretching and $\mathrm{CH}_{2}$ stretching, respectively. In the FT-IR spectra of PEG hydrogel-coated samples, the magnitude of the $\mathrm{C}=\mathrm{C}$ stretching band peak at $1640 \mathrm{~cm}^{-1}$ was decreased, which indicated the consumption of the double bonds during the photocrosslinking reaction. After the addition of acryl-PEG-RGDS conjugate, amide I

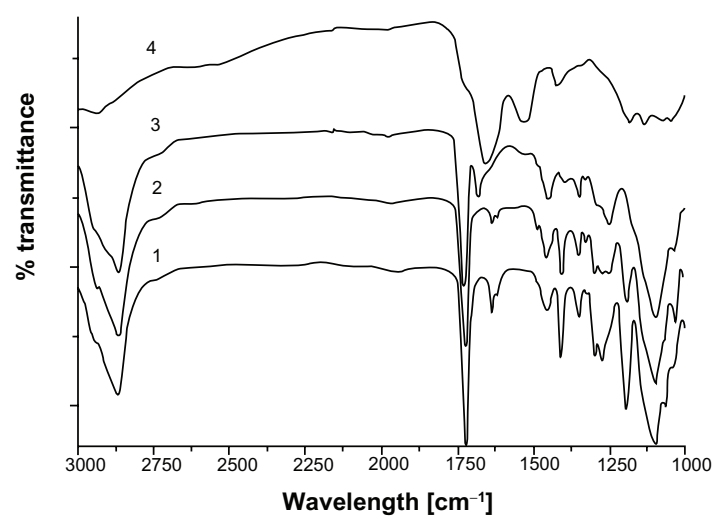

Figure 4 Fourier transform infrared spectra of (I) polyethylene glycol (PEG) diacrylate, (2) PEG hydrogel-coated magnetic iron oxide nanoparticles, (3) PEG hydrogel-coated magnetic iron oxide nanoparticles functionalized with arginineglycine-aspartic acid-serine, and (4) arginine-glycine-aspartic acid-serine. 
peak appeared at $1670 \mathrm{~cm}^{-1}$ - this peak was not observed in the remaining groups. The XRD pattern of PEG hydrogelcoated MIONPs agreed well with the initial crystal structure. Although the existence of amorphous PEG coating prevented detection of sharp peaks in the XRD, original magnetite peaks (seen in Figure 3) could be observed in the diffraction pattern (Figure S4A). Additionally, lyophilized PEG hydrogel-coated MIONPs responded well to an external magnet (Figure S4B).

Determination of the number of incorporated RGDS peptides per nanoparticle is technically challenging. ${ }^{46}$ Previously, Hern and Hubbell ${ }^{47}$ found a correlation between the percentage of acrylated peptide incorporation and acrylated peptide in PPS via radiolabeling assay. Using Hern and Hubbell's ${ }^{47}$ results and based on our experimental acryl-PEG-RGDS and PEGDA concentrations in PPS, it was found that $17.8 \%$ acryl-PEGRGDS is incorporated into the PEG hydrogel structure. This means that $0.1 \mathrm{mg} / \mathrm{mL}$ of biofunctional PEG hydrogel-coated MIONPs contains $2.6 \times 10^{-6} \mathrm{M}$ PEG-RGDS conjugate.

The change in hydrodynamic size after each modification step was characterized via DLS. Size distribution of MIONPs in all groups is shown in Figure 5A. Diameter of APTMS-coated MIONPs was $6.4 \mathrm{~nm}$. After eosin binding on MIONPs, particle diameter increased to $12.6 \mathrm{~nm}$. When eosin-bound MIONPs were added to PPS, hydrodynamic

A

Size distribution by number

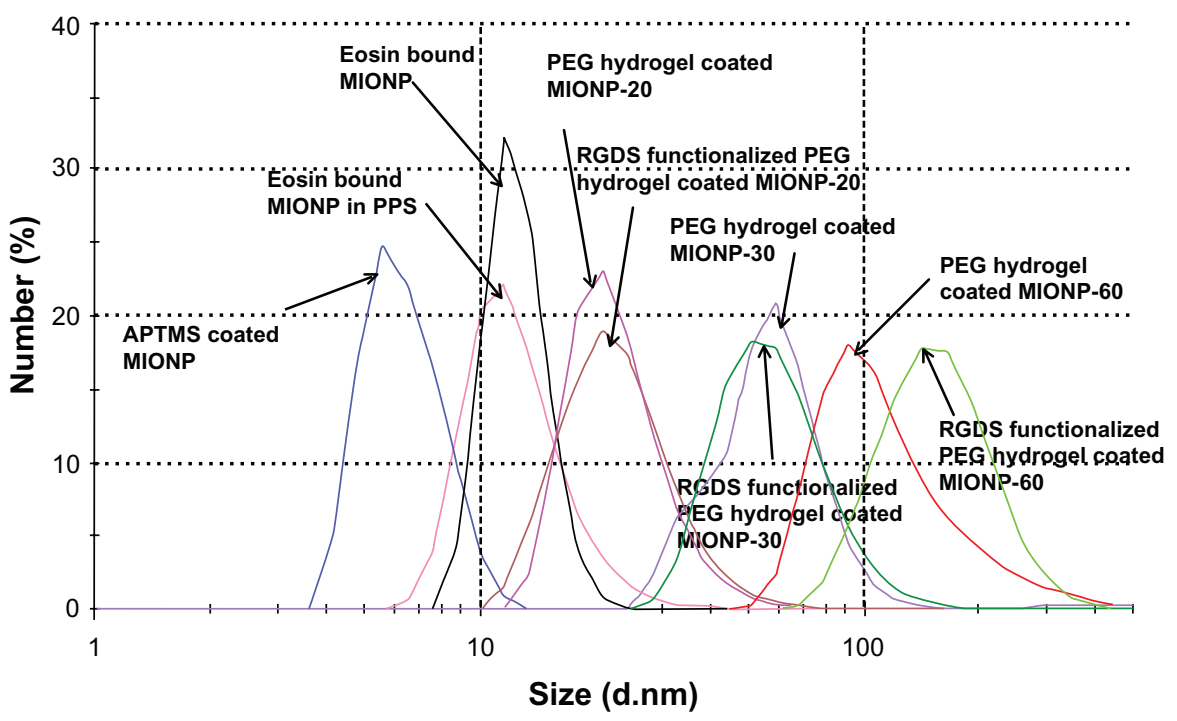

B

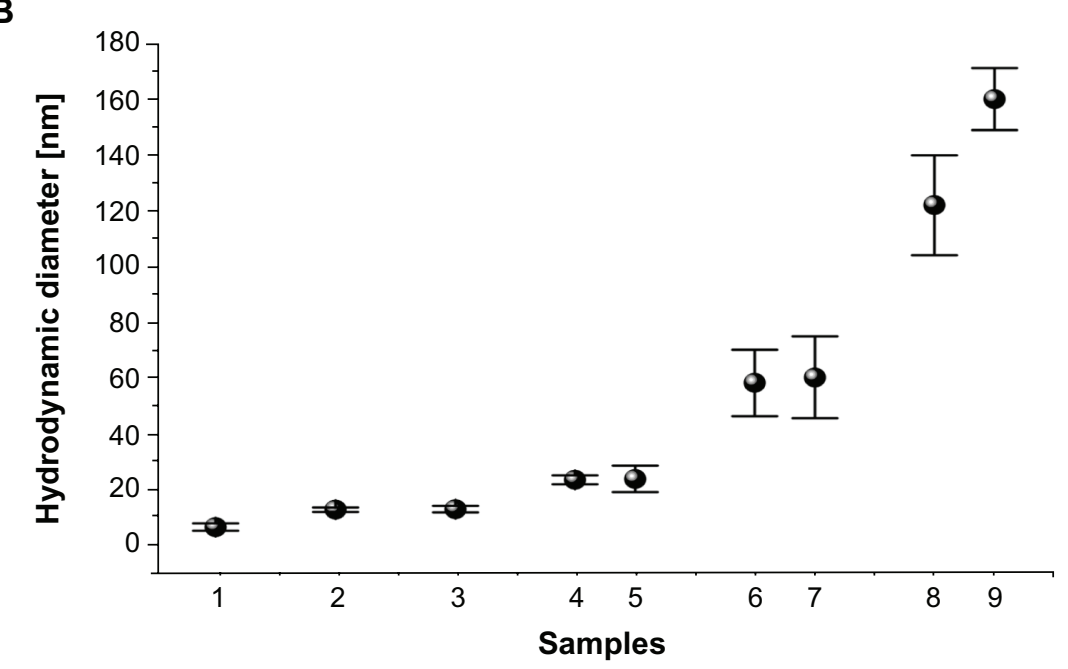

Figure 5 (A) Size distribution of various magnetic iron oxide nanoparticles (MIONPs) obtained via dynamic light scattering. (B) Change of hydrodynamic diameter before and after polyethylene glycol (PEG) hydrogel coating: (I) 3-aminopropylsilane (APTMS)-coated MIONPs, (2) eosin-bound MIONPs, (3) eosin-bound MIONPs in prepolymer solution (PPS), (4) PEG hydrogel-coated MIONP-20, (5) arginine-glycine-aspartic acid-serine (RGDS)-functionalized PEG hydrogel-coated MIONP-20, (6) PEG hydrogel-coated MIONP-30, (7) RGDS-functionalized PEG hydrogel-coated MIONP-30, (8) PEG hydrogel-coated MIONP-60, (9) RGDS-functionalized PEG hydrogel-coated MIONP-60. Note: Numbers in abbreviations MIONP-20, MIONP-30, MIONP-60 indicate corresponding illumination times in seconds. 
size was measured as $13.6 \mathrm{~nm}$. The diameter of particles increased as a function of irradiation time: PEG hydrogelcoated MIONPs at diameters of 23.3, 58, and $122 \mathrm{~nm}$ were obtained at 20, 30, and 60 seconds of laser exposure times, respectively. No significant change in the hydrodynamic size was observed with the incorporation of acryl-PEG-RGDS conjugate into the PEG hydrogel coating for samples irradiated for 20 and 30 seconds. The increase in hydrodynamic size after each step of coating is summarized in Figure 5B.

Zeta potential measurement results show changes in surface charge of MIONPs during each step of their coating (Figure 6). Initially, APTMS-coated MIONPs had a typical positive zeta potential. After negatively charged eosin was bound onto the surface of the MIONPs, the total charge of the nanoparticles was changed from positive $(22.3 \mathrm{mV})$ to almost neutral $(2.3 \mathrm{mV})$. PEG hydrogel-coated MIONPs became negatively charged (about $-30 \mathrm{mV}$ ), which is similar to the value obtained with pure PEGDA solution $(-28 \mathrm{mV})$. The addition of PEG-RGDS conjugate did not affect the surface charge significantly.

After the coating of MIONPs with PEG hydrogel, SEM and AFM were used to characterize the morphology of the MIONPs. Figure 7 shows a SEM image of MIONPs coated with PEG hydrogel and with a laser exposure time of 20 seconds. The particles are spherically shaped and have an average diameter of $35 \pm 12 \mathrm{~nm}$, which is consistent with the value measured with DLS. AFM height images (Figure S5A and $\mathrm{B}$ ) demonstrate that many nanoparticles were coated individually but several nanoparticles were encapsulated in clusters.

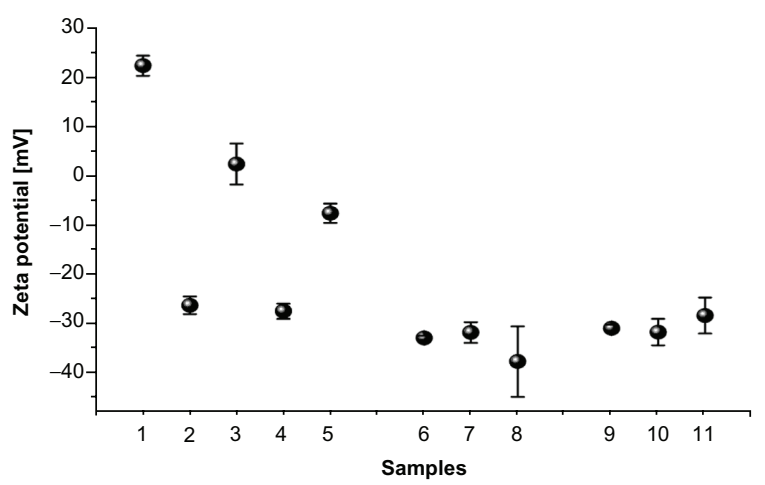

Figure 6 Zeta potential measurement before and after polyethylene glycol (PEG) hydrogel coating: (I) 3-aminopropylsilane-coated magnetic iron oxide nanoparticles (MIONPs), (2) eosin, (3) eosin-bound MIONPs, (4) PEG diacrylate, (5) eosinbound MIONPs in prepolymer solution, (6) PEG hydrogel-coated MIONP-20, (7) PEG hydrogel-coated MIONP-30, (8) PEG hydrogel-coated MIONP-60, (9) arginine-glycine-aspartic acid-serine (RGDS)-functionalized PEG hydrogel-coated MIONP-20, (I0) RGDS-functionalized PEG hydrogel-coated MIONP-30, (I I) RGDS functionalized PEG hydrogel-coated MIONP-60.

Note: Numbers in abbreviations MIONP-20, MIONP-30, MIONP-60 indicate corresponding illumination times in seconds.

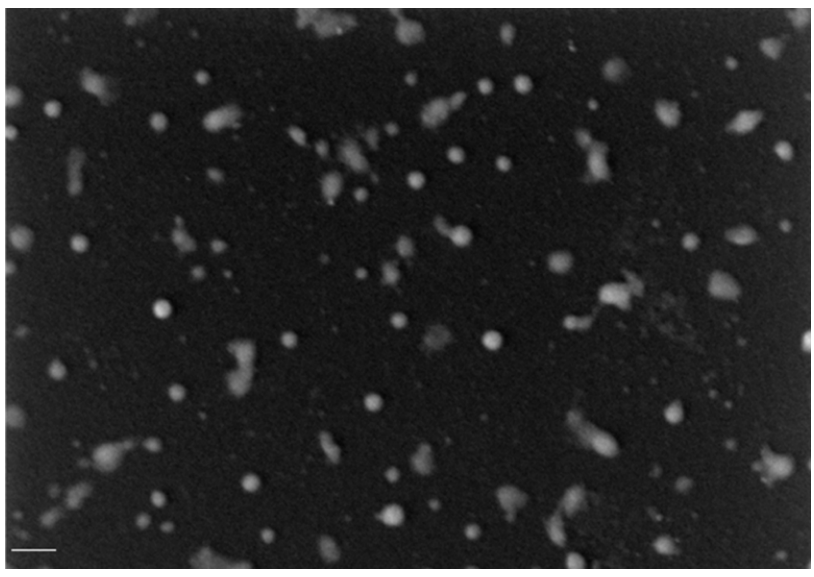

Figure 7 Microscopic characterization of polyethylene glycol hydrogel-coated magnetic iron oxide nanoparticles with an illumination period of 20 seconds: scanning electron microscopy image (scale bar: $200 \mathrm{~nm}$ ) (20 kV; magnification: $75 \mathrm{KX})$.

\section{Cytotoxicity and cellular uptake of MIONPs}

In order to evaluate the toxicity of coated MIONPs, the viability of HeLa cells was examined after 24 and 48 hours of treatment with MIONPs at concentrations of 0.01, 0.05, and $0.1 \mathrm{mg} / \mathrm{mL}$ (Figure 8). At low MIONP concentration $(0.01 \mathrm{mg} / \mathrm{mL})$, the viability was $>80 \%$ for cells treated with MIONPs in different groups. At higher concentrations (0.05 and $0.1 \mathrm{mg} / \mathrm{mL}$ ), samples of eosin-coated MIONPs and of MIONPs coated with PEG hydrogel and with 20 seconds of laser exposure time had a toxic effect on HeLa cells cultured for 24 and 48 hours. This was probably because of the presence of unreacted pendant groups in the system and could possibly be eliminated via repeated swelling and lyophilization of MIONPs. When laser exposure time for photopolymerization was increased from 20 seconds to 30 and 60 seconds, the PEG hydrogel coating did not affect cell viability significantly. The viability remained above $80 \%$ for cells in the groups that were treated with the highest nanoparticle concentration $(0.1 \mathrm{mg} / \mathrm{mL})$.

Prussian blue staining and ICP-OES assays were used to compare intracellular uptake of MIONPs in different groups. As shown in Figure S6, MIONPs coated with RGDS-functionalized PEG hydrogel and with 60 seconds of illumination time demonstrated a blue granule appearance stronger than the intensity of blue color observed in other groups. This indicates higher intracellular Fe concentration than the other conditions, with both APTMS or only PEG hydrogel-coated MIONPs. ICP-OES results also indicated a dramatic increase in the iron content of HeLa cells incubated with RGDSfunctionalized PEG hydrogel-coated MIONPs (60 seconds of illumination time) (Figure 9). RGDS-functionalized 


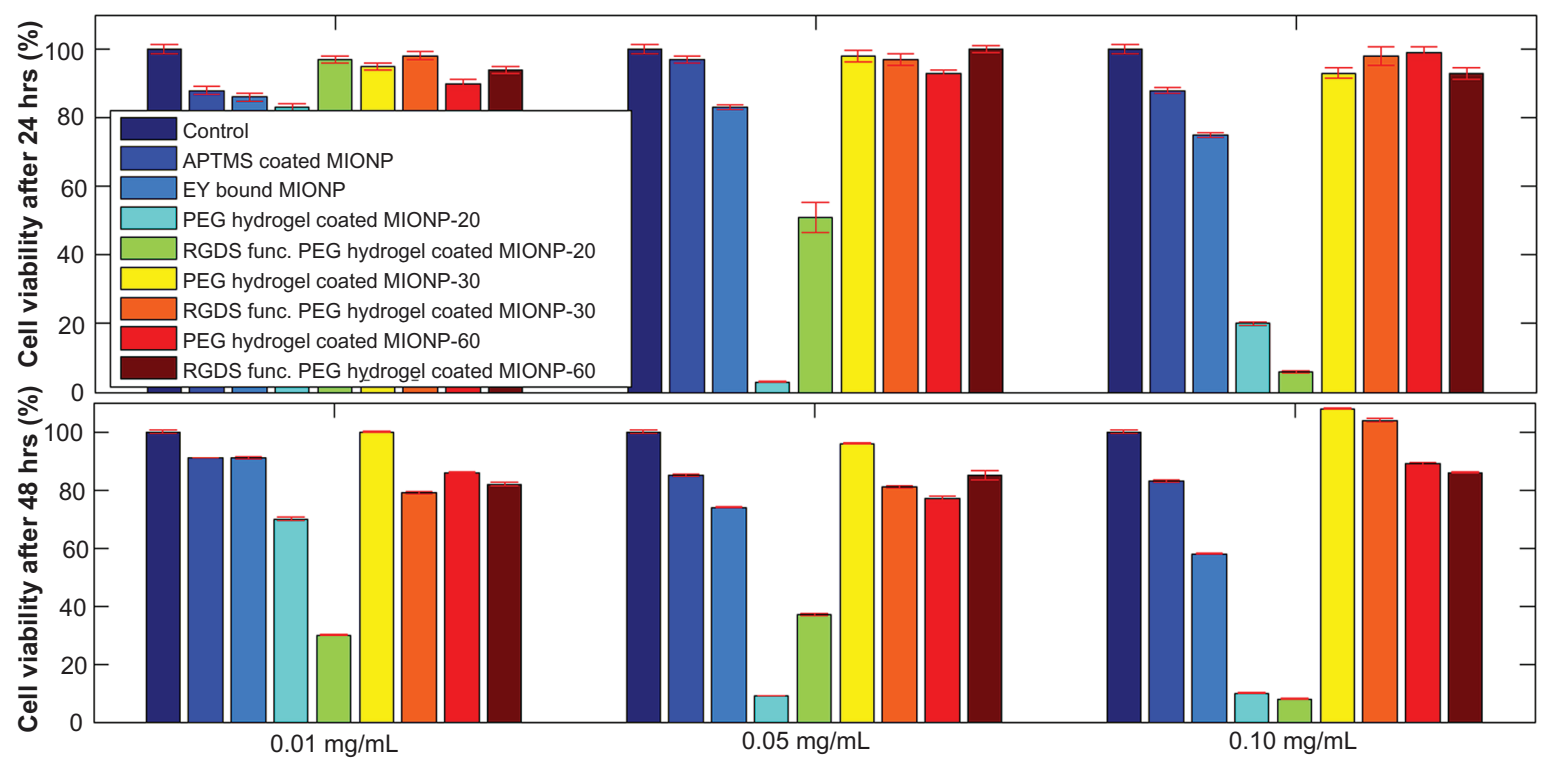

MIONP concentration in cell culture

Figure 8 Cytotoxicity profiles of varied magnetic iron oxide nanoparticles (MIONPs) after 24 and 48 hours of incubation with HeLa cells. Note: Numbers in abbreviations MIONP-20, MIONP-30, MIONP-60 indicate corresponding illumination times in seconds.

Abbreviations: APTMS, 3-aminopropylsilane; PEG, polyethylene glycol; RGDS, arginine-glycine-aspartic acid-serine.

PEG hydrogel coating with 60 seconds of illumination time increased iron internalization into HeLa cells from $3 \mathrm{pg}$ $\mathrm{Fe} / \mathrm{cell}$ to $50 \mathrm{pg} \mathrm{Fe/cell.}$

\section{Discussion}

Various strategies for coating MIONPs in order to prevent agglomeration and to increase blood circulation time have been developed using natural and synthetic polymers such as alginate, ${ }^{42}$ dextran, ${ }^{48}$ liposome, ${ }^{39}$ polyvinylpyrrolidone, ${ }^{12}$ and PEG. ${ }^{41,43}$ Some of the limitations involved in the existing approaches are lack of stabilization in body fluid, difficulty reaching targeted cell types, and difficulty retaining sufficient magnetic property. In order to address these limitations, starting from ultrasmall stable MIONPs, coating of particles with covalently cross-linked biofunctional PEG hydrogel has been investigated in this study. From the particle perspective, size of the biofunctional PEG hydrogel-coated MIONPs can be adjusted by changing the laser illumination time, and hydrodynamic size can be kept within "small size" regimen. The in vitro results of this study show that coating of nanoparticles using surface-initiated photopolymerization of PEGDA does not compromise viability of HeLa cells. Further, biofunctionalization of PEG hydrogel coating with RGDS using an illumination time of 60 seconds significantly increases the efficiency of uptake of MIONPs by HeLa cells.

Recent studies have demonstrated that a PEG coating minimizes the intracellular uptake of nanoparticles by macrophages ${ }^{49}$ and increases circulation time in blood. ${ }^{50}$
In this work, MIONPs were coated with cross-linked PEG hydrogel in order to improve stability and achieve high water content around nanoparticles. Furthermore, the use of PEG hydrogel systems allows for a flexible skeleton for nanoparticle biofunctionalization, because a specific ligand or therapeutic agent can be incorporated into PEG hydrogel during photopolymerization with this technique. Peptides that bind to the cell surface are functional tools for targeting the vascular endothelium in solid tumors for specific delivery of therapeutic and imaging agents. ${ }^{36}$ Thus, covalent conjugation of cell adhesion peptides to PEG hydrogel is a promising method for solving obstacles such as transportation and internalization of MIONPs into targeted specific cell types. Therefore, in the present study, PEG hydrogel was functionalized via acryl-PEG-RGDS conjugate in order to enhance accumulation and internalization of MIONPs into cancer cells. The conjugation reaction between peptide and PEG was carried out successfully, and was verified by FT-IR spectroscopy (Figure S1).

The XRD pattern of APTMS-coated MIONPs confirms their crystallinity (Figure 3A). As expected, the blackbrown aqueous suspension of these particles responded to an external magnetic field generated by a handheld magnet (Figure 3B). The photoinitiator eosin is covalently bound on APTMS-coated MIONPs with 57\% efficiency using WRK chemistry. UV-Vis spectra and FT-IR spectra of both APTMS-coated and eosin-bound MIONPs confirmed the amidation reaction between the carboxyl group of eosin 


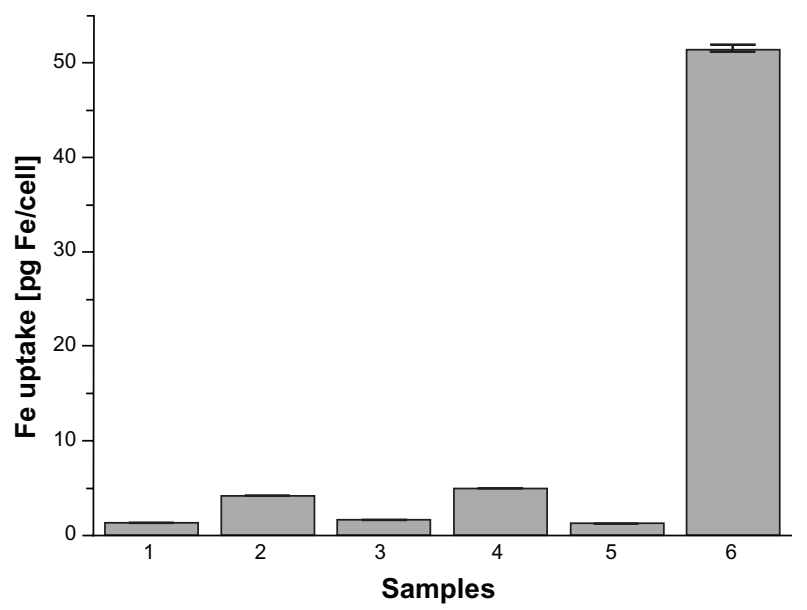

Figure 9 Uptake of magnetic iron oxide nanoparticles (MIONPs) by HeLa cells after 24 hours of incubation, as measured by inductively coupled plasma optical emission spectrometry: (I) control, (2) 3-aminopropylsilane-coated MIONPs, (3) polyethylene glycol (PEG) hydrogel-coated MIONP-30, (4) PEG hydrogel-coated MIONP-30 functionalized with arginine-glycine-aspartic acid-serine, (5) PEG hydrogel-coated MIONP-60, and (6) PEG hydrogel-coated MIONP-60 functionalized with arginine-glycine-aspartic acid-serine.

Note: Numbers in abbreviations MIONP-20, MIONP-30, and MIONP-60 indicate corresponding illumination times in seconds.

and amine groups of APTMS-coated MIONPs (Figure S2A and B). Acryl-PEG-RGDS conjugate and eosin-bound MIONPs were added to the PPS and reacted via photopolymerization at mild conditions at different time intervals in order to tune the hydrogel thickness around MIONPs. According to the FT-IR spectra in Figure 4, the peaks for $\mathrm{C}-\mathrm{O}-\mathrm{C}, \mathrm{CH}_{2}$, and $\mathrm{C}-\mathrm{H}$ gave strong evidence about the surface coverage of MIONPs with PEG hydrogel. Further, the FT-IR spectrum for the RGDS-functionalized PEG hydrogel-coated MIONPs demonstrated conjugation of acryl-PEG-RGDS conjugate within the cross-linked structure, which resulted in a biofunctional PEG hydrogel (Figure 4). Figure S4A shows PEG hydrogel-coated MIONPs retained their magnetic properties. The freeze-dried form of PEG hydrogel-coated particles could respond to an external magnetic field generated by a handheld magnet as expected (Figure S4B). Hence, PEG hydrogel did not disrupt the magnetic strength of the iron oxide nanoparticles. Protection of magnetic field property was also observed in previous studies performed with PEG coating of MIONPs. ${ }^{14,41}$ In addition, bulk encapsulation of MIONPs within PEG hydrogel was studied by Meenach et $\mathrm{al}^{22}$ and magnetic property was shown to be preserved.

Pharmacokinetics and cellular uptake of nanoparticles are primarily based on physicochemical features such as hydrodynamic size, surface charge, and morphology. ${ }^{51}$ In the present study, hydrodynamic size of nanoparticles before and after the coating steps was characterized by
DLS, which is based on analyzing the diffusion behavior of the nanoparticles in solution. ${ }^{52}$ Figure $5 \mathrm{~A}$ and $\mathrm{B}$ shows that increasing the illumination time also increased the thickness of the PEG hydrogel coating around the MIONPs - narrow size distribution could be obtained for nanoparticles in different groups. In addition, biofunctionalization of PEG hydrogel with the addition of acryl-PEG-RGDS into the PPS did not change the coating thickness or particle size distribution significantly. The electrostatic interactions of the nanoparticles could be characterized by their surface charge, which is analyzed via zeta potential measurement. ${ }^{53}$ The surface charge of the MIONPs before and after the coating steps was characterized via zeta potential measurement (Figure 6). The results obtained from DLS and zeta potential measurement suggest that PEG hydrogel-coated MIONPs can have extended stability in aqueous solution as a result of the electrostatic repulsion between negatively charged nanoparticles. The zeta potential results also suggest that PEG hydrogel coating may prevent aggregation of these nanoparticles during circulation in blood. The surface topography of PEG hydrogel-coated MIONPs was characterized via SEM (Figure 7) and AFM (Figure S5). Comparison of AFM height (Figure S5A and B) and phase images (Figure S5C and D) shows the core-shell structure of nanoparticles. The particles had an inner magnetite core and an outer polymeric shell of PEG, which provides additional evidence for the presence of PEG hydrogel on the surface of MIONPs. It was observed that some nanoparticles were encapsulated in clusters. Encapsulation of nanoparticles in groups could not be completely eliminated because the nanoparticles were tightly packed within the PPS. Size and surface modification of nanoparticles could result in a different response of cells to nanoparticles in terms of nonspecific or targeted cellular uptake. ${ }^{54}$ With the method presented here, it is possible to control the thickness and cross-link density of the hydrogel coating around nanoparticles through laser illumination time and/or monomer concentration. Further, various ligands or biomolecules could be covalently conjugated into a PEG hydrogel coating, which may also allow for specific cell targeting.

Toxicity is an important property to consider when evaluating the performance of nanoparticles for biomedical applications. ${ }^{3,55}$ Cytotoxic agents could compromise the viability of many cells within 48 hours of treatment. ${ }^{55}$ In this study, the viability of HeLa cells was characterized by exposing the cells to nanoparticles in different groups. Figure 8 shows that the PEG hydrogel coating of nanoparticles at laser illumination times of 30 and 60 seconds, with or 
without RGDS, did not affect cell viability significantly for either 24 or 48 hours of treatment.

The ability to deliver effective concentrations of therapeutic agents into the tumor site is a significant step towards the diagnosis and treatment of tumor or cancer tissue. ${ }^{56}$ The ligand-receptor approach provides medical intervention to influence cancer cells only, not the healthy cells, depending on the molecular identification system. ${ }^{56}$ This approach also has great potential to enhance the efficiency of therapeutic and contrast agents, by increasing the amount of those agents around the target site and by decreasing systemic administration, which reduces or prevents cytotoxicity or background signal imaging. Also, this method may identify the tumor at an earlier phase than the currently existing approaches and eliminate an invasive biopsy requirement.

In order to demonstrate whether intracellular uptake of biofunctional PEG hydrogel-coated nanoparticles by cancer cells will be affected by the coating, the authors used Prussian blue staining and ICP-OES measurements after 24 hours of incubation of HeLa cells with nanoparticles (Figures 9 and S6). Figure 9 demonstrates that RGDS-functionalized PEG hydrogel-coated MIONPs with 60 seconds of laser illumination time had a 17-fold higher uptake by the HeLa cells than that of APTMS-coated MIONPs. These results suggest that RGDS was critical for cellular uptake and that PEG hydrogel formed with 60 seconds of laser illumination time was optimal for the biocompatibility of MIONPs at high concentration. Comparison of the cytotoxicity and cellular uptake results of PEG hydrogel-coated MIONPs with 60 seconds of laser illumination time suggested that the PEG coating prevented interaction between MIONPs and cells and that the RGDS-functionalized PEG hydrogel coating provided internalization of MIONPs into cells. Based on viability results, it was observed that RGDS-functionalized PEG hydrogel-coated nanoparticles affected cell viability minimally at the highest nanoparticle concentration $(0.1 \mathrm{mg} / \mathrm{mL})$ and they displayed the highest cellular uptake. After biodegradation of MIONPs, cells could be exposed to excessive free iron. Free iron accumulation at high levels results in toxicity. ${ }^{57}$ The cross-linked structure of PEG coating may allow for slow diffusion of iron ions after biodegredation. This feature of PEG hydrogel can prevent high iron exposure in tissues of the target area. Therefore, an RGDS-functionalized PEG hydrogel coating may not only enhance internalization of nanoparticles but also prevent accumulation of excessive free iron by means of its crosslinked network.
RGDS functionalization of the PEG hydrogel coating around nanoparticles also has a potential use in hyperthermia therapy, which involves raising the local temperature at the tumor site to promote cell death when MIONPs are exposed to an external magnetic field. ${ }^{6,19,58,59}$ In order to kill cancer cells efficiently by heating, this technique requires optimal particle size and tumor targeting. Nanoparticles must be fast, specific, and effective to reach the target site and to be taken up by tumor cells. ${ }^{60}$ Their performance in vivo is restricted by several factors such as aggregation, short half-life in blood circulation, inefficient cellular uptake, and nonspecific targeting. ${ }^{60}$ The size of MIONPs coated with biocompatible materials should be controlled in order to overcome difficulties involved in their transport and internalization by specific cells. Based on the results obtained in this study, the biofunctional PEG hydrogel coating of MIONPs offers a promising platform for in vivo applications. Not only may this approach allow specific tissues in the body to be targeted but also it may reduce the quantity of diagnostic or therapeutic agents necessary for achieving a particular concentration within the surroundings of the target tissue.

\section{Conclusion}

MIONPs have enormous potential for the early detection of cancer or tumor and for targeted therapies. In order to overcome the limitations associated with MIONP technologies, the authors have utilized a novel strategy of surfaceinitiated photopolymerization to coat nanoparticles with RGDS-functionalized PEG hydrogel. The coating of MIONPs within biofunctional PEG hydrogel resulted in increased uptake by HeLa cells and increased viability of cells compared to the uptake and viability measured in control groups. Not only this method is applicable to the coating of iron oxide nanoparticles but it can also be extended to encapsulate nanoparticles with different geometries, such as nanorods, or chemistry, such as fullerenes. The strategy used in this study to coat nanoparticles may also be a significant tool for diagnostic and therapeutic imaging technologies to utilize for increasing blood circulation time and achieving the targeted delivery of drugs.

\section{Acknowledgments}

This study was supported by the College of Engineering at Koç University, Turkey, and by a Marie Curie international Reintegration Grant (FP7-IRG-239471) to SK. The authors would like to thank Dr Ugur Unal, Dr Ozgur Birer, Selcuk Acar, Ibrahim Hocaoglu, and Huseyin Enis Karahan for their help with XRD, SEM, ICP-OES, DLS, and AFM experiments. XRD and SEM analyses were performed 
at the Koç University Surface Science and Technology Center.

\section{Disclosure}

The authors report no conflicts of interest in this work.

\section{References}

1. Sun C, Lee JS, Zhang M. Magnetic nanoparticles in MR imaging and drug delivery. Adv Drug Deliv Rev. 2008;60(11):1252-1265.

2. Mahmoudi M, Simchi A, Imani M. Recent advances in surface engineering of superparamagnetic iron oxide nanoparticles for biomedical applications. J Iran Chem Soc. 2010;7(Suppl 1):S1-S27.

3. Ai J, Biazar E, Jafarpour M, et al. Nanotoxicology and nanoparticle safety in biomedical designs. Int J Nanomedicine. 2011;6:1117-1127.

4. Saltan N, Kutlu HM, Hür D, Işcan A, Say R. Interaction of cancer cells with magnetic nanoparticles modified by methacrylamido-folic acid. Int J Nanomedicine. 2011;6:477-484.

5. Hanini A, Schmitt A, Kacem K, Chau F, Ammar S, Gavard J. Evaluation of iron oxide nanoparticle biocompatibility. Int $J$ Nanomedicine. 2011;6:787-794.

6. Oh JK. Iron oxide-based superparamagnetic polymeric nanomaterials: design, preparation, and biomedical application. Prog Polym Sci. 2011; 36(1):168-189.

7. Mou Y, Hou Y, Chen B, et al. In vivo migration of dendritic cells labeled with synthetic superparamagnetic iron oxide. Int J Nanomedicine. 2011;6:2633-2640.

8. Xie Y, Zeng P, Siegel RA, Wiedmann TS, Hammer BE, Longest PW. Magnetic deposition of aerosols composed of aggregated superparamagnetic nanoparticles. Pharm Res. 2010;27(5):855-865.

9. Hu F, Neoh KG, Cen L, Kang ET. Cellular response to magnetic nanoparticles "PEGylated" via surface-initiated atom transfer radical polymerization. Biomacromolecules. 2006;7(3):809-816.

10. Gupta AK, Gupta M. Synthesis and surface engineering of iron oxide nanoparticles for biomedical applications. Biomaterials. 2005;26(18): 3995-4021.

11. Cole AJ, David AE, Wang J, Galbán CJ, Hill HL, Yang VC. Polyethylene glycol modified, cross-linked starch-coated iron oxide nanoparticles for enhanced magnetic tumor targeting. Biomaterials. 2011;32(8):2183-2193.

12. Lee HY, Lim NH, Seo JA, et al. Preparation and magnetic resonance imaging effect of polyvinylpyrrolidone-coated iron oxide nanoparticles. J Biomed Mater Res B Appl Biomater. 2006;79(1):142-150.

13. Acar HYC, Garaas RS, Syud F, Bonitatebus P, Kulkarni AM. Superparamagnetic nanoparticles stabilized by polymerized PEGylated coatings. J Magn Magn Mater. 2005;293(1):1-7.

14. Tong S, Hou S, Zheng Z, Zhou J, Bao G. Coating optimization of superparamagnetic iron oxide nanoparticles for high $\mathrm{T} 2$ relaxivity. Nano Lett. 2010;10(11):4607-4613.

15. Barbucci R. Hydrogels: Biological Properties and Applications. New York, NY: Springer; 2009.

16. Zhu J. Bioactive modification of poly(ethylene glycol) hydrogels for tissue engineering. Biomaterials. 2010;31(17):4639-4656.

17. Peng XH, Qian X, Mao H, et al. Targeted magnetic iron oxide nanoparticles for tumor imaging and therapy. Int $J$ Nanomedicine. 2008;3(3):311-321.

18. Kizilel S, Pérez-Luna VH, Teymour F. Photopolymerization of poly(ethylene glycol) diacrylate on eosin-functionalized surfaces. Langmuir. 2004;20(20):8652-8658.

19. Gupta AK, Naregalkar RR, Vaidya VD, Gupta M. Recent advances on surface engineering of magnetic iron oxide nanoparticles and their biomedical applications. Nanomedicine (Lond). 2007;2(1):23-39.

20. Burdick JA, Anseth KS. Photoencapsulation of osteoblasts in injectable RGD-modified PEG hydrogels for bone tissue engineering. Biomaterials. 2002;23(22):4315-4323.
21. Cruise GM, Hegre OD, Lamberti FV, et al. In vitro and in vivo performance of porcine islets encapsulated in interfacially photopolymerized poly(ethylene glycol) diacrylate membranes. Cell Transplant. 1999; 8(3): 293-306.

22. Meenach SA, Hilt JZ, Anderson KW. Poly(ethylene glycol)-based magnetic hydrogel nanocomposites for hyperthermia cancer therapy. Acta Biomater. 2010;6(3):1039-1046.

23. Kizilel S, Scavone A, Liu X, et al. Encapsulation of pancreatic islets within nano-thin functional polyethylene glycol coatings for enhanced insulin secretion. Tissue Eng Part A. 2010;16(7):2217-2228.

24. Xiang Y, Peng Z, Chen D. A new polymer/clay nano-composite hydrogel with improved response rate and tensile mechanical properties. Eur Polym J. 2006;42(9):2125-2132.

25. Schexnailder P, Schmidt G. Nanocomposite polymer hydrogels. Colloid Polym Sci. 2009;287(1):1-11.

26. Meenach SA, Anderson KW, Hilt JZ. Hydrogel nanocomposites: biomedical applications, biocompatibility, and toxicity analysis. In: Webster TJ, editor. Safety of Nanoparticles: From Manufacturing to Medical Applications, 1st ed. New York, NY: Springer; 2009:131-158.

27. Degirmenbasi N, Kalyon DM, Birinci E. Biocomposites of nanohydroxyapatite with collagen and poly(vinyl alcohol). Colloids Surf $B$ Biointerfaces. 2006;48(1):42-49.

28. Frimpong RA, Fraser S, Hilt JZ. Synthesis and temperature response analysis of magnetic-hydrogel nanocomposites. J Biomed Mater Res A. 2007;80(1):1-6.

29. Meenach SA, Anderson AA, Suthar M, Anderson KW, Hilt JZ. Biocompatibility analysis of magnetic hydrogel nanocomposites based on poly(N-isopropylacrylamide) and iron oxide. J Biomed Mater Res A. 2009;91(3):903-909.

30. Okada M, Yasuda S, Kimura T, et al. Optimization of amino group density on surfaces of titanium dioxide nanoparticles covalently bonded to a silicone substrate for antibacterial and cell adhesion activities. $J$ Biomed Mater Res A. 2006;76(1):95-101.

31. Kizilel S, Sawardecker E, Teymour F, Pérez-Luna VH. Sequential formation of covalently bonded hydrogel multilayers through surface initiated photopolymerization. Biomaterials. 2006;27(8):1209-1215.

32. Kizilel S, Pérez-Luna VH, Teymour F. Mathematical model for surfaceinitiated photopolymerization of poly(ethylene glycol) diacrylate. Macromol Theor Simul. 2006;15(9):686-700.

33. Kizilel S, Pérez-Luna VH, Teymour F. Modeling of PEG hydrogel membranes for biomedical applications. Macromol React Eng. 2009; 3(5-6):271-287.

34. Kizilel S. Mathematical model for microencapsulation of pancreatic islets within a biofunctional PEG hydrogel. Macromol Theor Simul. 2010;19(8-9):514-531.

35. Zhang C, Jugold M, Woenne EC, et al. Specific targeting of tumor angiogenesis by RGD-conjugated ultrasmall superparamagnetic iron oxide particles using a clinical 1.5-T magnetic resonance scanner. Cancer Res. 2007;67(4):1555-1562.

36. Kessler T, Bieker R, Padró T, et al. Inhibition of tumor growth by RGD peptide-directed delivery of truncated tissue factor to the tumor vasculature. Clin Cancer Res. 2005;11(17):6317-6324.

37. Kim HC, Lee SK, Lee SW, Jeong SW. A reactive polythiophene for protein immobilization. Polym Adv Technol. 2009;20(3):298-302.

38. Porjazoska A, Yilmaz OK, Baysal K, et al. Synthesis and characterization of poly(ethylene glycol)-poly(D,L-lactide-co-glycolide) poly(ethylene glycol) tri-block co-polymers modified with collagen: a model surface suitable for cell interaction. J Biomater Sci Polym Ed. 2006;17(3): 323-340.

39. Liao Z, Wang H, Lv R, et al. Polymeric liposomes-coated superparamagnetic iron oxide nanoparticles as contrast agent for targeted magnetic resonance imaging of cancer cells. Langmuir. Epub February 22, 2011.

40. Váró G, Brown LS, Needleman R, Lanyi JK. Binding of calcium ions to bacteriorhodopsin. Biophys J. 1999;76(6):3219-3226.

41. Gupta AK, Wells S. Surface-modified superparamagnetic nanoparticles for drug delivery: preparation, characterization, and cytotoxicity studies. IEEE Trans Nanobioscience. 2004;3(1):66-73. 
42. Ma HL, Qi XR, Maitani Y, Nagai T. Preparation and characterization of superparamagnetic iron oxide nanoparticles stabilized by alginate. Int J Pharm. 2007;333(1-2):177-186.

43. Park JY, Daksha P, Lee GH, Woo S, Chang Y. Highly water-dispersible PEG surface modified ultra small superparamagnetic iron oxide nanoparticles useful for target-specific biomedical applications. Nanotechnology. 2008;19(36):365603.

44. Mikhaylova M, Kim DK, Berry CC, et al. BSA immobilization on amine-functionalized superparamagnetic iron oxide nanoparticles. Chem Mater. 2004;16(12):2344-2354.

45. Stuart B. Infrared Spectroscopy: Fundamentals and Applications. Hoboken, NJ: John Wiley \& Sons; 2004.

46. Xie J, Chen K, Lee HY, et al. Ultrasmall c(RGDyK)-coated Fe3O4 nanoparticles and their specific targeting to integrin alpha(v)beta3-rich tumor cells. J Am Chem Soc. 2008;130(24):7542-7543.

47. Hern DL, Hubbell JA. Incorporation of adhesion peptides into nonadhesive hydrogels useful for tissue resurfacing. J Biomed Mater Res. 1998;39(2):266-276.

48. Moore A, Marecos E, Bogdanov A Jr, Weissleder R. Tumoral distribution of long-circulating dextran-coated iron oxide nanoparticles in a rodent model. Radiology. 2000;214(2):568-574.

49. Zhang Y, Kohler N, Zhang M. Surface modification of superparamagnetic magnetite nanoparticles and their intracellular uptake. Biomaterials. 2002;23(7):1553-1561.

50. Gref R, Minamitake Y, Peracchia MT, Trubetskoy V, Torchilin V, Langer R. Biodegradable long-circulating polymeric nanospheres. Science. 1994;263(5153):1600-1603.

51. Veiseh O, Gunn JW, Zhang M. Design and fabrication of magnetic nanoparticles for targeted drug delivery and imaging. Adv Drug Deliv Rev. 2010;62(3):284-304.
52. Wang YX, Hussain SM, Krestin GP. Superparamagnetic iron oxide contrast agents: physicochemical characteristics and applications in MR imaging. Eur Radiol. 2001;11(11):2319-2331.

53. Ge Y, Zhang Y, Xia J, et al. Effect of surface charge and agglomerate degree of magnetic iron oxide nanoparticles on $\mathrm{KB}$ cellular uptake in vitro. Colloids Surf B Biointerfaces. 2009;73(2):294-301.

54. Wu X, Tan Y, Mao H, Zhang M. Toxic effects of iron oxide nanoparticles on human umbilical vein endothelial cells. Int J Nanomedicine. 2010;5:385-399.

55. Lewinski N, Colvin V, Drezek R. Cytotoxicity of nanoparticles. Small. 2008;4(1):26-49.

56. Koo YE, Reddy GR, Bhojani M, et al. Brain cancer diagnosis and therapy with nanoplatforms. Adv Drug Deliv Rev. 2006;58(14):1556-1577.

57. Jain TK, Reddy MK, Morales MA, Leslie-Pelecky DL, Labhasetwar V. Biodistribution, clearance, and biocompatibility of iron oxide magnetic nanoparticles in rats. Mol Pharm. 2008;5(2):316-327.

58. Silva AC, Oliveira TR, Mamani JB, et al. Application of hyperthermia induced by superparamagnetic iron oxide nanoparticles in glioma treatment. Int J Nanomedicine. 2011;6:591-603.

59. Rodríguez-Luccioni H, Latorre-Esteves M, Méndez-Vega J, et al. Enhanced reduction in cell viability by hyperthermia induced by magnetic nanoparticles. Int J Nanomedicine. 2011;6:373-380.

60. Hofmann-Amtenbrink M, Hofmann H. Superparamagnetic nanoparticles for biomedical applications. In: Tan MC, editor. Nanostructured Materials for Biomedical Applications, 1st ed. Kerala, India: Transworld Research Network; 2009. 


\section{Supplementary figures}

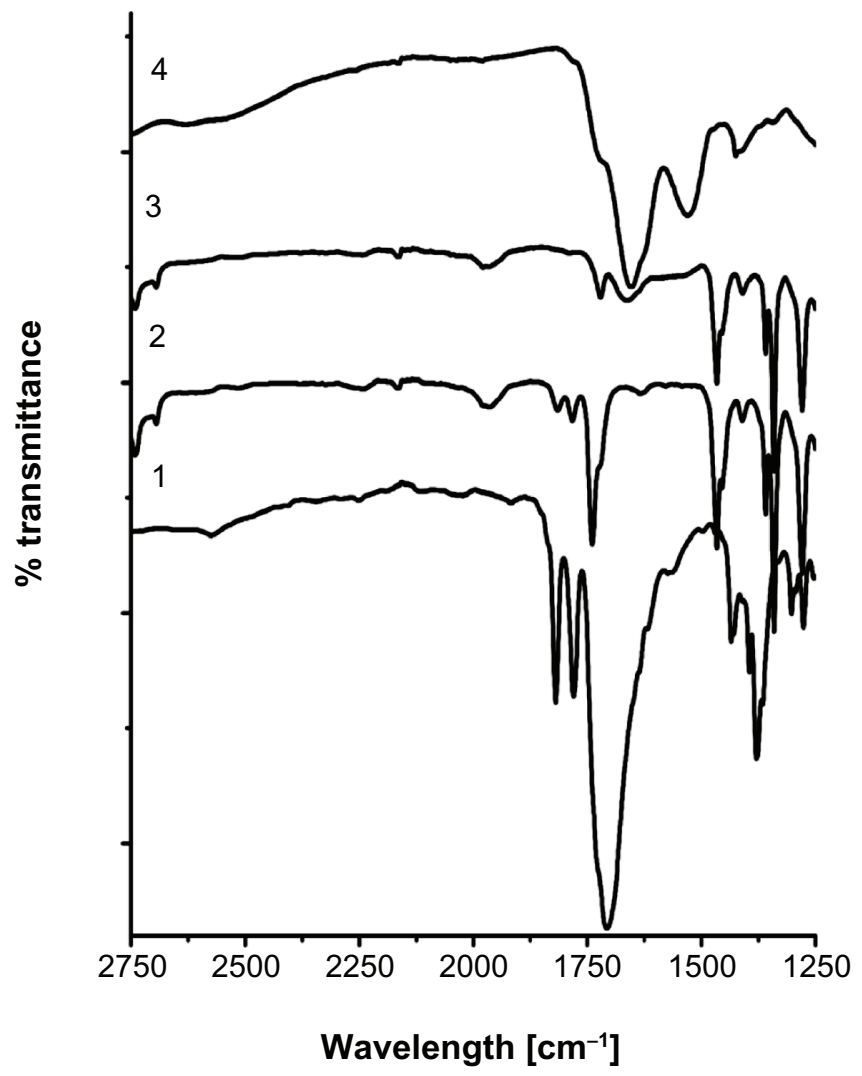

Figure SI Fourier transform infrared spectra of (I) acrylic acid N-hydroxysuccinimide, (2) acrylate-polyethylene glycol-N-hydroxysuccinimide, (3) acrylate-polyethylene glycolarginine-glycine-aspartic acid-serine, and (4) arginine-glycine-aspartic acid-serine. 
A

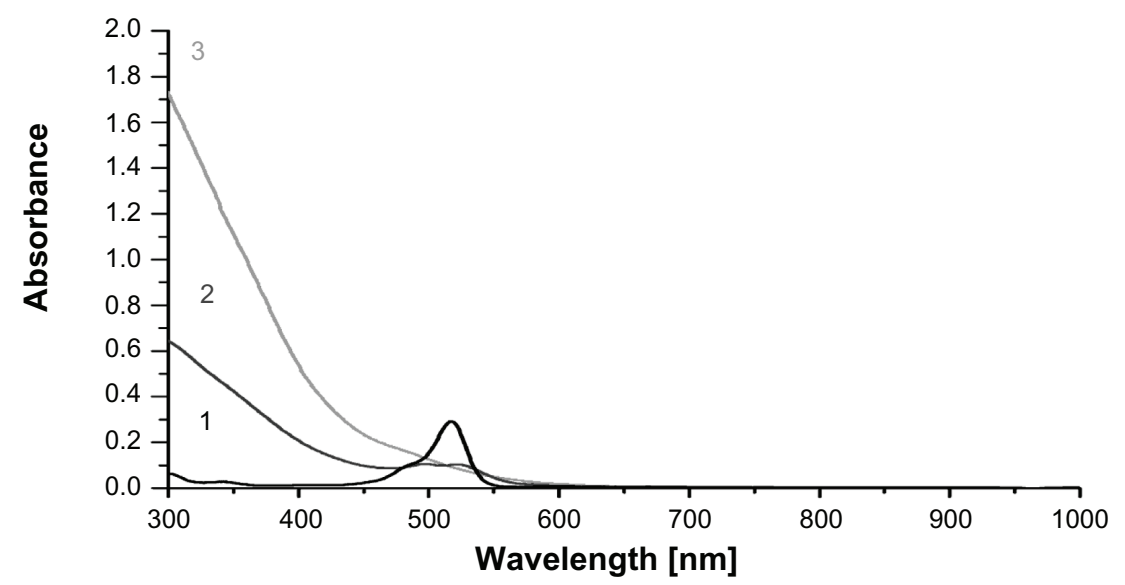

B

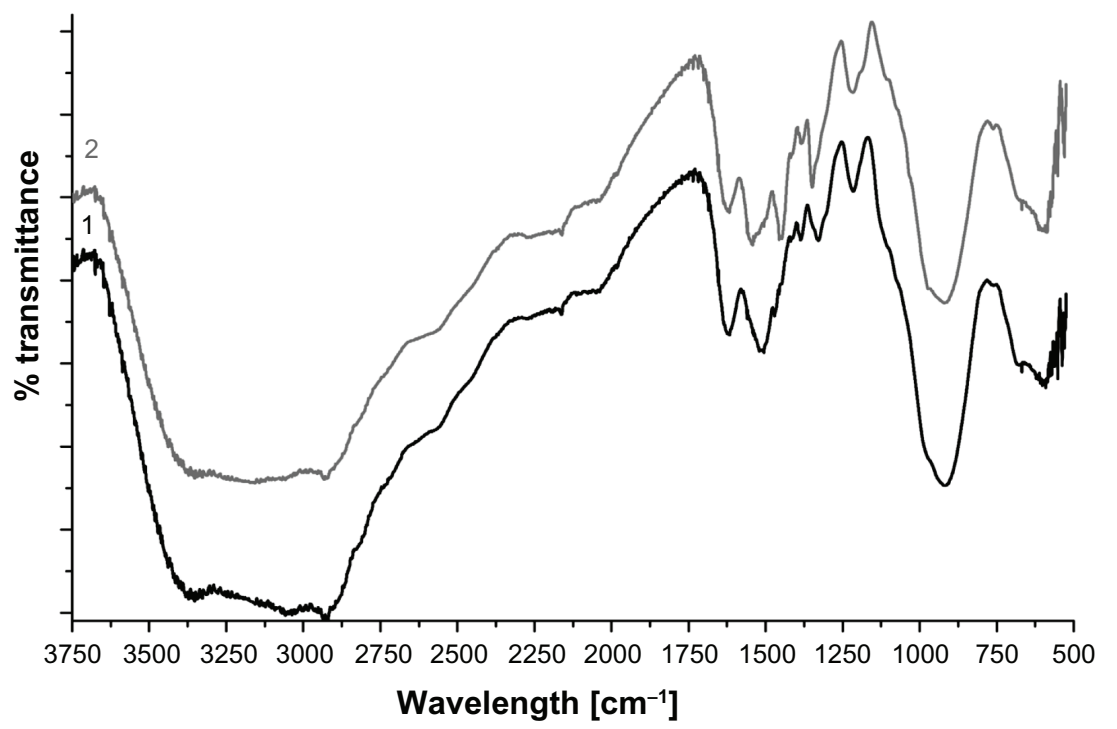

Figure S2 (A) Ultraviolet and visible spectra of (I) eosin only, (2) eosin-bound magnetic iron oxide nanoparticles (MIONPs), and (3) 3-aminopropylsilane-coated MIONPs; (B) Fourier transform infrared spectra of (I) 3-aminopropylsilane-coated MIONPs and (2) eosin-bound MIONPs.

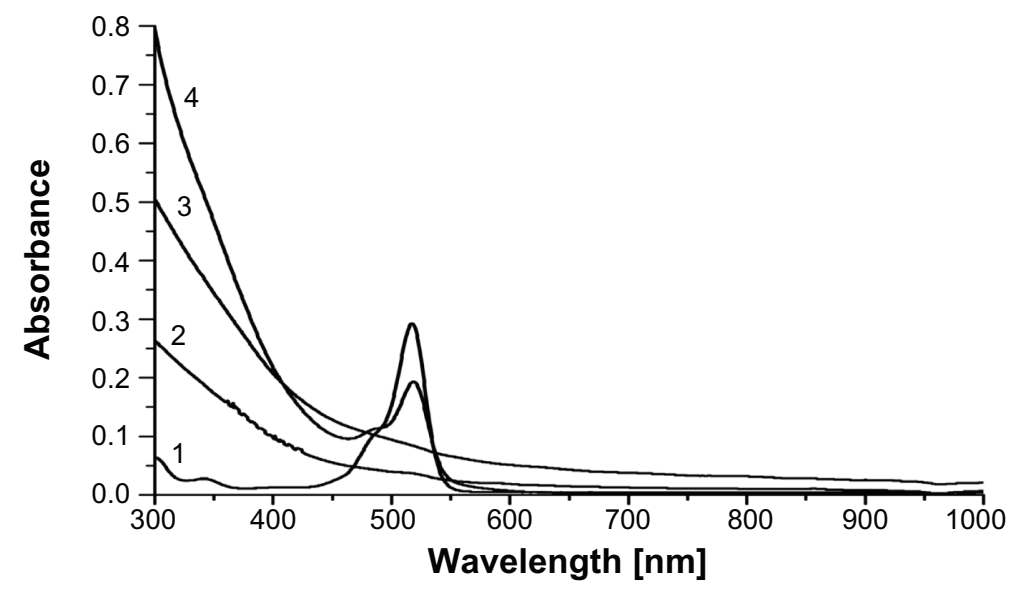

Figure S3 Photobleaching of eosin after photopolymerization: (1) eosin only; (2) arginine-glycine-aspartic acid-serine-functionalized polyethylene glycol hydrogel-coated magnetic iron oxide nanoparticles (MIONPs); (3) polyethylene glycol hydrogel-coated MIONPs; (4) eosin-bound MIONPs in prepolymer solution before photopolymerization reaction. 


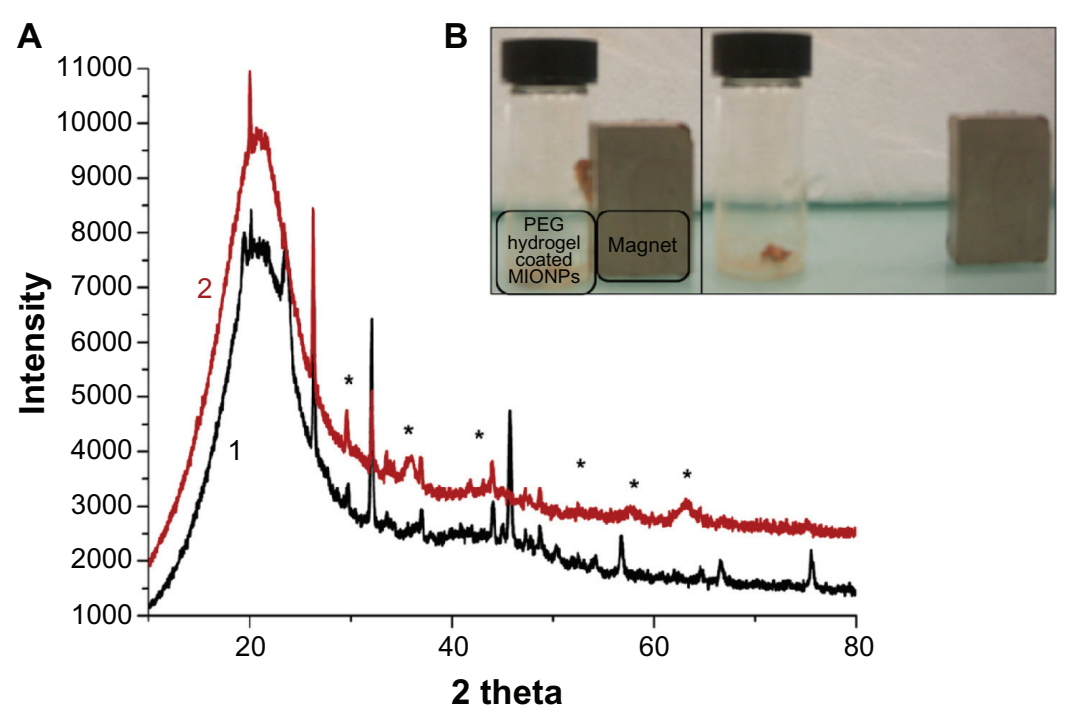

Figure S4 (A) X-ray diffraction patterns of (I) lyophilized polyethylene glycol (PEG) hydrogel and (2) lyophilized PEG hydrogel-coated magnetic iron oxide nanoparticles (MIONPs); (B) lyophilized PEG hydrogel-coated MIONPs under the magnetic field of a magnet.

A

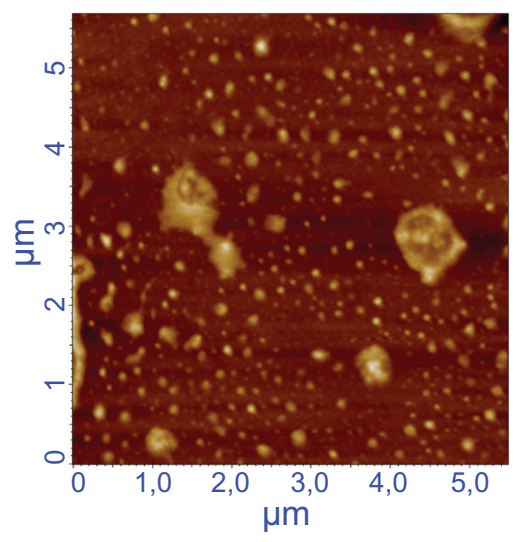

C

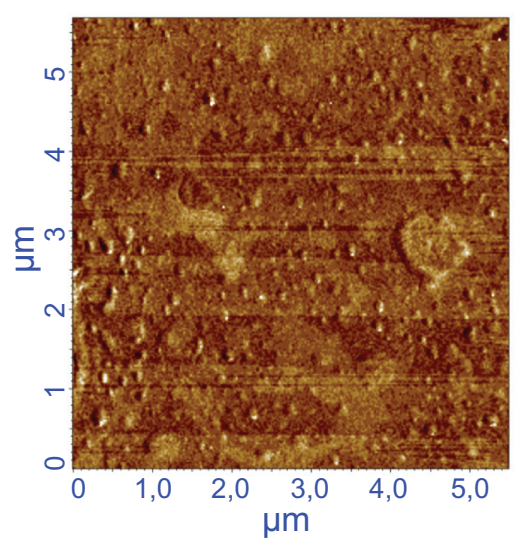

B
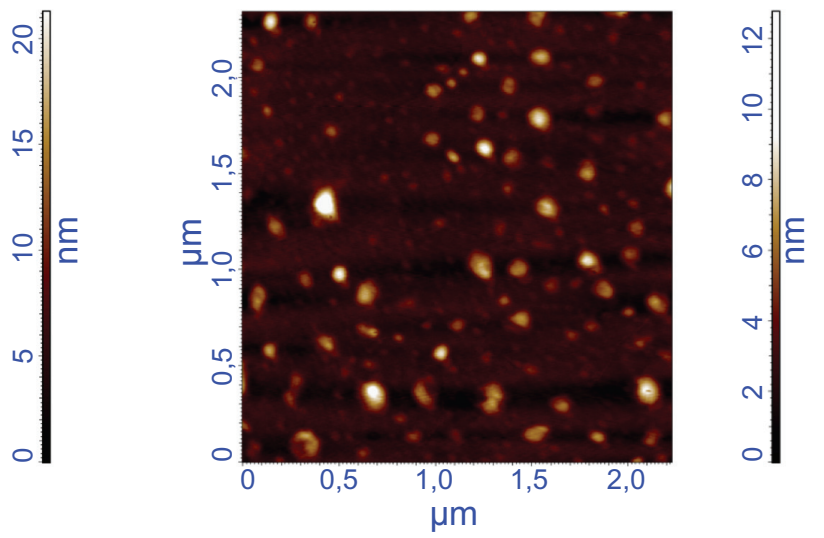

D
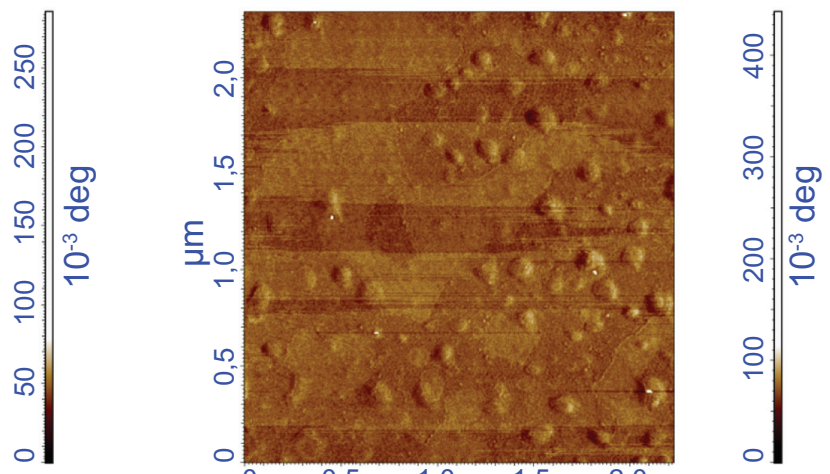

Figure S5 (A) Atomic force microscopy (AFM) height image of a $5 \mu \mathrm{m}^{2}$ area; (B) AFM height image of a $2 \mu \mathrm{m}^{2}$ area; (C) AFM phase image of a $5 \mu \mathrm{m}^{2}$ area; and (D) AFM phase image of a $2 \mu \mathrm{m}^{2}$ area. 
A

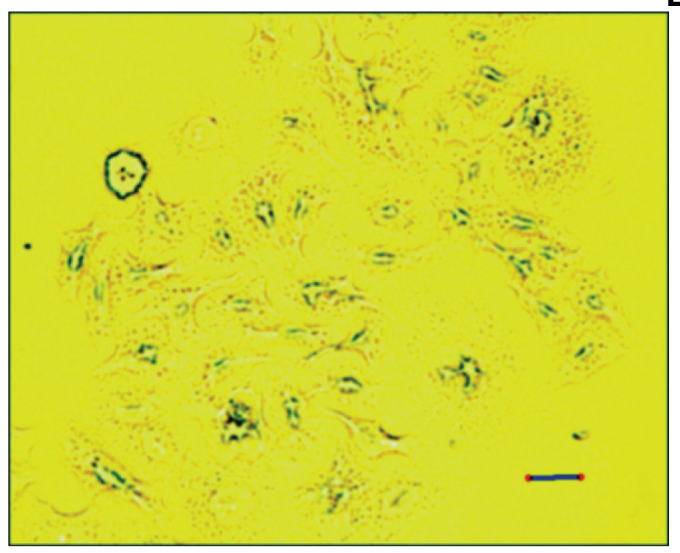

B

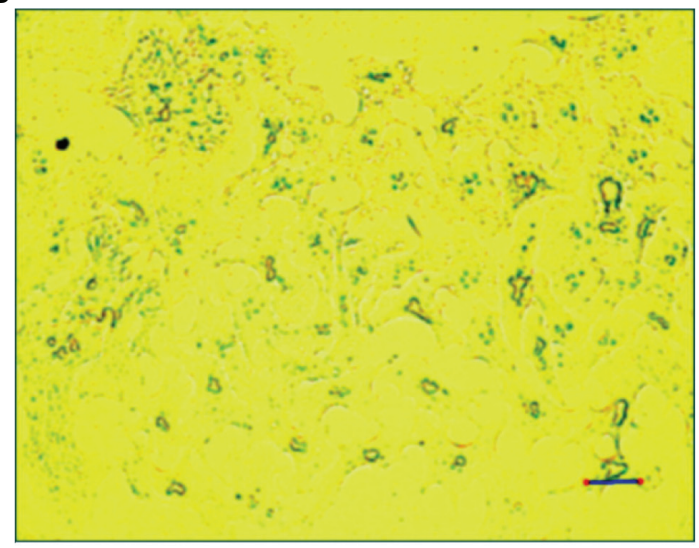

C

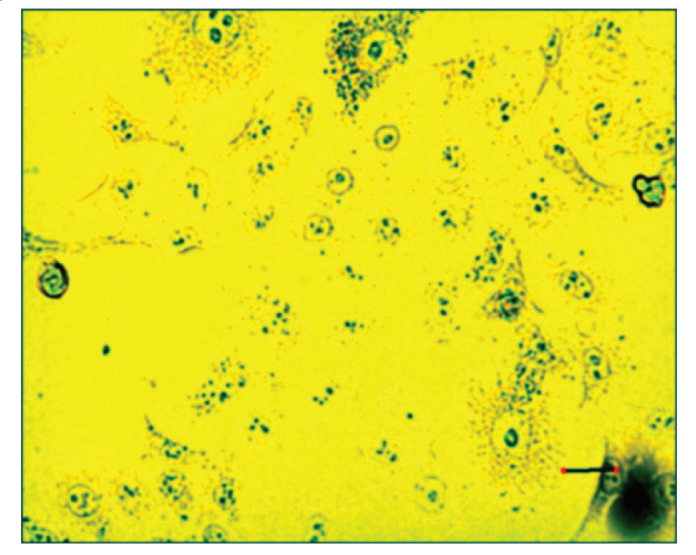

D

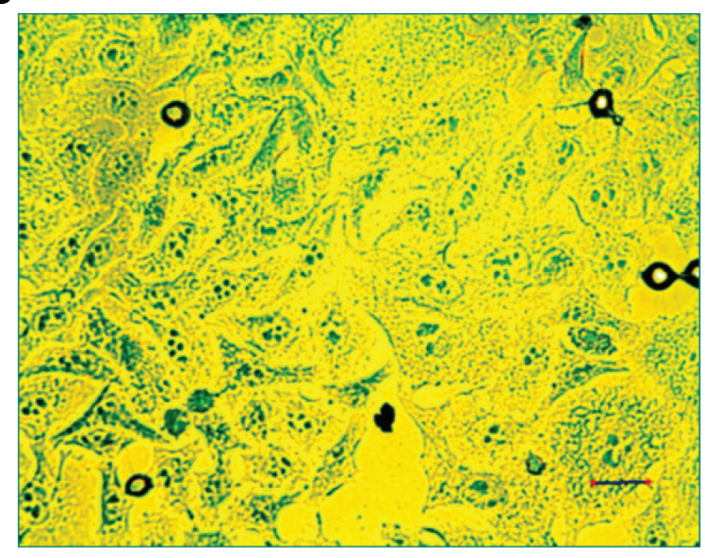

Figure S6 HeLa cells stained with Prussian blue: (A) control; (B) 3-aminopropylsilane-coated magnetic iron oxide nanoparticles (MIONPs); (C) polyethylene glycol hydrogelcoated MIONP-60; (D) arginine-glycine-aspartic acid-serine-functionalized polyethylene glycol hydrogel-coated MIONP-60 (scale bar: $25 \mu \mathrm{M}$ ).

Note: Number in abbreviation MIONP-60 indicates corresponding illumination time in seconds.

International Journal of Nanomedicine

\section{Publish your work in this journal}

The International Journal of Nanomedicine is an international, peerreviewed journal focusing on the application of nanotechnology in diagnostics, therapeutics, and drug delivery systems throughout the biomedical field. This journal is indexed on PubMed Central, MedLine, CAS, SciSearch ${ }^{\circledR}$, Current Contents ${ }^{\circledR} /$ Clinical Medicine,

\section{Dovepress}

Journal Citation Reports/Science Edition, EMBase, Scopus and the Elsevier Bibliographic databases. The manuscript management system is completely online and includes a very quick and fair peer-review system, which is all easy to use. Visit http://www.dovepress.com/ testimonials.php to read real quotes from published authors. 